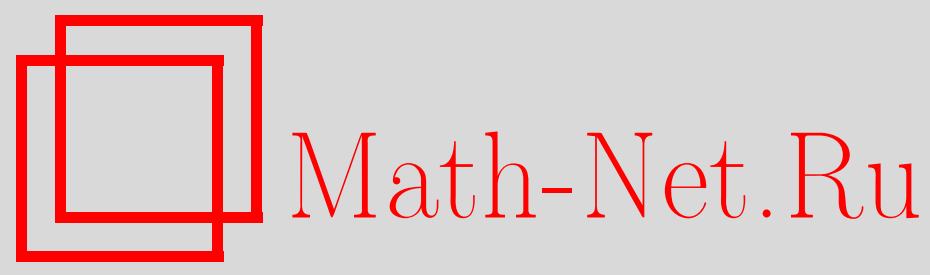

Е. П. Вдовин, Максимальные порядки абелевых подгрупп в конечных группах Шевалле, Матем. заметки, 2001, том 69, выпуск 4, 524-549

DOI: https://doi.org/10.4213/mzm521

Использование Общероссийского математического портала Math-Net.Ru подразумевает, что вы прочитали и согласны с пользовательским соглашением http://www . mathnet.ru/rus/agreement

Параметры загрузки:

IP : 18.209 .158 .208

26 апреля 2023 г., 10:14:09

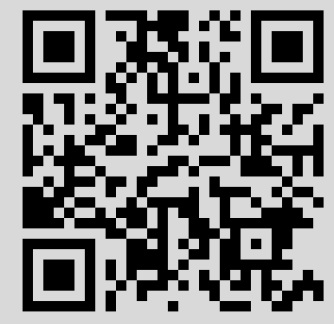




\title{
МАКСИМАЛЬНЫЕ ПОРЯДКИ АБЕЛЕВЫХ ПОДГРУПП В КОНЕЧНЫХ ГРУППАХ ШЕВАЛЛЕ
}

\section{Е. П. В довин}

\begin{abstract}
В настоящей работе для любой конечной группы $G$ лиева типа (кроме $\left.{ }^{2} F_{4}(q)\right)$ найден порядок $a(G)$ большой абелевой подгруппы или даны его нижняя и верхняя оценки (в группах $F_{4}(q), E_{6}(q), E_{7}(q), E_{8}(q)$ и $\left.{ }^{2} E_{6}\left(q^{2}\right)\right)$. В тех группах, в которых число $a(G)$ найдено точно, большая абелева подгруппа совпадает с большой унипотентной или большой полупростой абелевой подгруппой. В группах $F_{4}(q), E_{6}(q), E_{7}(q)$, $E_{8}(q)$ и ${ }^{2} E_{6}\left(q^{2}\right)$ доказано, что если абелева подгруппа содержит нецентральный полупростой элемент, то ее порядок меньше порядка некоторой абелевой унипотентной группы. Поэтому в этих группах большие абелевы подгруппы унипотентны, и для того чтобы найти значение чисел $a(G)$ в этих группах, необходимо найти порядки больших унипотентных абелевых подгрупп. Таким образом, доказано, что в любой конечной группе лиева типа (кроме $\left.{ }^{2} F_{4}(q)\right)$ большая абелева подгруппа является большой унипотентной или большой полупростой абелевой подгруппой.
\end{abstract}

Библиографоия: 21 название.

1. Введение. В данной работе изучаются строение и порядки больших абелевых подгрупп в конечных группах Шевалле. Различные специальные абелевы подгрупшы в конечных группах лиева типа изучались многими авторами. Так, например, Картер [1], [2] получил строение связных централизаторов в алгебраических группах над алгебраически замкнутьм полем характеристики $p>0$, а также строение и порядки их подгрупп неподвижных точек относительно автоморфизма Ф робениуса. В частности, он нашел порядки максимальных торов во всех конечных классических односвязньх группах Шевалле. Изучение порядков связных централизаторов в исключительных группах Шевалле закончил Деризиотис в своих работах [3], [4]. В нескольких последовательных статьях [5]-[8] Барри и Уонг получили порядки и строение больших унипотентных абелевых подгрупп в конечных классических групах Шевалле.

В настоящей работе для любой конечной группы $G$ лиева типа (кроме $\left.{ }^{2} F_{4}(q)\right)$ найден порядок $a(G)$ большой абелевой подгруппы или дана его нижняя и верхняя оценка (в группах $F_{4}(q), E_{6}(q), E_{7}(q), E_{8}(q)$ и $\left.^{2} E_{6}\left(q^{2}\right)\right)$. В тех группах, в которых число $a(G)$ найдено точно, большая абелева подгрупа совпадает с большой унипотентной или большой полупростой абелевой подгруппой. В группах $F_{4}(q), E_{6}(q), E_{7}(q), E_{8}(q)$ и ${ }^{2} E_{6}\left(q^{2}\right)$ доказано, что если абелева подгруппа содержит нецентральньй полупростой элемент,

Работа вьполнена при поддержке ФЦП “Интеграция", проект № 274, Российского фонда фундаментальных исследований, грант № 99-01-00550, и СО РАН, грант для коллективов молодых ученых, постановление Президиума № 83 от 10.03.2000. 
то ее порядок меньше порядка некоторой абелевой унипотентной группы. Поэтому в этих группах большие абелевы подгрупш унипотентны, и для того, чтобы найти значение чисел $a(G)$ в этих групшах, необходимо найти порядки больших унипотентных абелевых подгрупп.

Обозначения и определения, используемые в данной работе, можно найти в [9]-[11]. Если $\varphi$ - гомоморфизм групшы $G, g$ - элемент групшы $G$, то $G^{\varphi}, g^{\varphi}$ - образы групшы $G$ и элемента $g$ относительно гомоморфизма $\varphi$ соответственно.

Обозначения, связанные с конечными групшами лиева типа, выбраны так же, как в [10]. Под групшой Шевалле, если не оговорено противное, понимается как универсальная группа Шевалле, так и любая ее факторгруппа по подгруппе из центра. При изучении групп Шевалле $G F(q)$ будет обозначать поле порядка $q, p$ - его характеристику, $G F(q)^{*}$ - мультипликативную группу поля $G F(q), K=\overline{G F(q)}$ - алгебраическое замькание поля $G F(q)$. Группа Шевалле, соответствующая корневой системе $\Phi$ над полем $G F(q)$, обозначается через $\Phi(q)$. Групша Вейля, соответствующая корневой системе $\Phi$, обозначается $W(\Phi)$. Скрученные группы будут обозначаться символами ${ }^{2} A_{n}\left(q^{2}\right)$, ${ }^{2} D_{n}\left(q^{2}\right),{ }^{2} E_{n}\left(q^{2}\right),{ }^{3} D_{4}\left(q^{3}\right),{ }^{2} B_{2}(q),{ }^{2} G_{2}(q)$ и ${ }^{2} F_{4}(q) ; \Phi^{+}\left(\Phi^{-}\right)$обозначает множество положительных (отрищательньх) корней корневой системы $\Phi, \Delta=\left\{r_{1}, \ldots, r_{k}\right\}$ - множество фундаментальных корней, причем нумерация выбирается, как в [10, формула (3.4)]. Элемент групшы Шевалле $\Phi(q)$ назьвается полупростым, если его порядок взаимно прост с $p$, и унипотентным, если его порядок является степенью $p$. Аналогично, nолупростая и унипотентная подгруппы групш $\Phi(q)$ определяются как подгруппы, порядок которых взаимно прост с $p$ ( $p^{\prime}$-подгруппы) и является степенью $p$ соответственно. Расширенной диаграммой Дынкина группы Шевалле назьвается диаграмма, которая получается после добавления к исходной диаграмме Дынкина корня $-r_{0}$ (здесь $r_{0}$ - корень наибольшего веса) и присоединения его к остальньгм вершинам по обычному правилу. Расширенные диаграммы Дьнкина для всех типов корневых систем приведены в [3].

Для конечной группы $G$ через $a(G)$ обозначен максимум порядков ее абелевых подгрупп. Если $G$ - конечная группа лиева типа, то через $a_{u}(G)$ и $a_{s}(G)$ обозначены максимальные порядки унипотентных абелевых и полупростых абелевых подгрупп соответственно. Множество абелевых подгрупп из $G$, порядок которых совпадает с $a(G)$, $a_{u}(G)$ или $a_{s}(G)$, будут обозначаться через $A(G), A_{u}(G)$ или $A_{s}(G)$ соответственно. Элементы множеств $A(G), A_{u}(G)$ и $A_{s}(G)$ будем называть большими абелевыми, большими унипотентными абелевыми и большими полупростыми абелевыми подгрупами соответственно.

Автор выражает искреннюю благодарность Мазурову Виктору Даниловичу за полезные консультации и ценные замечания. Автор также благодарен Васильеву Андрею Викторовичу за чтение и обсуждение рукописи, которое позволило исправить многие ошибки и неточности первоначального варианта. Автор благодарит рецензента за полезные замечания.

2. Вспомогательные результаты о линейных алгебраических группах. В этом разделе будут приведены необходимые сведения о строении линейных алгебраических групп, а также будут получены вспомогательные утверждения, которые мы используем для получения оценок порядков абелевых подгрупп. Основные определения и результаты о строении и свойствах линейных алгебраических групп можно найти в [11]. Если $G$ - линейная алгебраическая группа, то через $G^{0}$ обозначена компонента единицы 
группы $G$. Линейная алгебраическая группа назьвается полупростой, если ее радикал тривиален, и линейная алгебраическая группа назьвается редуктивной, если ее унипотентный радикал тривиален; в обоих случаях не предполагается, что линейная алгебраическая группа связна. Хорошо известно (см., например, [11]), что связная полупростая линейная алгебраическая группа - это центральное произведение связных простых линейных алгебраических групп, а связная редуктивная линейная алгебраическая группа $G$ - это центральное произведение тора $S$ и полупростой группы $M$, причем $S=Z(G)^{0}$, $M=[R, R]$ и групша $S \cap M$ конечна.

Пусть $G$ - связная редуктивная линейная алгебраическая группа, $T$ - ее максимальный тор (под тором всегда понимается связная диагонализируемая группа), $B$ - ее подгрупа Бореля, содержащая тор $T$. В $G$ существует такая борелевская подгрупа $B^{-}$, что $B \cap B^{-}=T$. Пусть $\Phi-$ корневая система группы $G$ относительно тора $T$, $\varphi: N_{G}(T) \rightarrow N_{G}(T) / T=W-$ канонический гомоморфизм на групу Вейля $W$ группы $G, X_{\alpha}(\alpha \in \Phi)$ - корневые подгрупшы относительно тора $T$ (одномерные $T$-инвариантные унипотентные подгрупш групп $B$ и $B^{-}$). Действиегруппы Вейля $W$ на корневой системе $\Phi$ определяется следующим образом $[11$, п. 24.1]. Пусть для каждого элемента $w$ из $W$ зафиксирован некоторьй его представитель $n_{w}$ из группы $G$. Тогда группа Вейля действует на корнях корневой системы $\Phi$ по правилу $\alpha^{w}(t)=\alpha\left(t^{n_{w}}\right)$ для всех $\alpha \in \Phi, t \in T$. Хорошо известно, что $B=T U$, где $U=\left\langle X_{\alpha}: \alpha \in \Phi^{+}\right\rangle$- максимальная унипотентная подгруппа группы $G$, а $B^{-}=T U^{-}$, где через $U^{-}$обозначена группа $\left\langle X_{\alpha}: \alpha \in \Phi^{-}\right\rangle$. Если на $\Phi^{+}$задать порядок, то любой элемент из $U$ единственньп образом записьвается в виде произведения элементов из корневых подгрупп $U_{\alpha}$ (взятых в заданном порядке).

Любой элемент групшы $G$ единственным образом представим в виде $u n_{w} t v$, где $v \in U$, $t \in T, u \in U \cap n_{w} U^{-} n_{w}^{-1}$ (см., например, [11, теорема 28.3]). Такое представление элементов группы $G$ назьвается их разложением Брюа.

Пусть $G$ - связная простая алгебраическая группа, $\pi$ - ее некоторое точное рациональное представление, $\Gamma_{\pi}$ - решетка, порожденная весами представления $\pi$. Через $\Gamma_{a d}$ обозначается решетка, порожденная корнями системы $\Phi$, через $\Gamma_{s c}$ - решетка, порожденная фундаментальными весами. Решетки $\Gamma_{s c}, \Gamma_{\pi}$ и $\Gamma_{a d}$ не зависят от конкретного представления группы $G$, и справедливы следующие включения $\Gamma_{a d} \leqslant \Gamma_{\pi} \leqslant \Gamma_{s c}$.

Известно, что для корневой системы данного типа существует несколько различных простых алгебраических групп, которые называются изогениями. Они различаются строением групшы $\Gamma_{\pi}$ и порядком конечного центра. В том случае, когда решетка $\Gamma_{\pi}$ совпадает с $\Gamma_{s c}$, говорят, что группа $G$ односвязна, и она обозначается через $G_{s c}$. Ecли решетка $\Gamma_{\pi}$ совпадает с $\Gamma_{a d}$, то говорят, что группа $G$ имеет присоединенньй тип, и она обозначается через $G_{a d}$. Любая линейная алгебраическая группа с корневой системой $\Phi$ получается как факторгрупша групш $G_{s c}$ по подгруппе из ее центра. Центр группы $G_{a d}$ тривиален, и она проста как абстрактная группа.

Пусть $c_{i}$ - коэффициент, с которым фундаментальный корень $r_{i}$ входит в разложение корня $r_{0}$. Простые числа, делящие коэффищиенты $c_{i}$, назьваются плохими простыми числами.

Далее напомним фундаментальный результат о строении алгебраических групा.

Лемма 2.1 [11, пा. 21.3, 22.2]. Пусть $G$ - связная линейная алгебраическая групnа. Тогда все подгруппы Бореля группы $G$ сопряжены. Более того, максимальные торы и максимальные связные унипотентные подгруппы в $G$ - это в точнос- 
ти максимальные торы и максимальные связные унипотентные подгруппь групп Бореля. Кроме того, все максимальные торы и максимальные связные унипотентные подаруппы сопряжены и любой полупростой (соответственно унипотентный) әлемент лехит в некотором максимальном торе (соответственно максимальной связной унипотентной подгруппе).

Теперь напомним, каким образом связаны конечные групы лиева типа и простые линейные алгебраические группы. Пусть $G$ - связная простая линейная алгебраическая группа, определенная над алгебраически замкнутьм полем характеристики $p>0, \sigma-$ эндоморфизм группы $G$ такой, что множество его неподвижных точек $G_{\sigma}$ конечно. Эндоморфизм $\sigma$ с таким условием в дальнейшем будет называться автоморфизмом Фробениуса, хотя он может и не совпадать с классическим автоморфизмом Фробениуса. Отметим, что $\sigma$ является автоморфизмом, если группа $G$ рассматривается как абстрактная групша, и $\sigma$ является эндоморфизмом, если $G$ рассматривается как алгебраическая группа. В общем случае $\sigma$ имеет вид $q \sigma_{0}$, где $q=p^{\alpha}$ - возведение в $q$-ю степень, а $\sigma_{0}-$ графовьй автоморфизм порядка 1,2 или 3 . Тогда $O^{p^{\prime}}\left(G_{\sigma}\right)$ - группа лиева типа над конечным полем характеристики $p$, и любую группу лиева типа (нормальную или скрученную) можно получить таким образом. Далее $\sigma$ всегда будет обозначать некоторьй автоморфизм $ф$ робениуса.

Пусть $T$ - максимальньй $\sigma$-инвариантньй тор связной простой алгебраической группы $G$. В дальнейшем максимальным тором группы $G_{\sigma}\left(\right.$ соответственно $\left.O^{p^{\prime}}\left(G_{\sigma}\right)\right)$ будем назьвать группу вида $T_{\sigma}$ (соответственно $T_{\sigma} \cap O^{p^{\prime}}\left(G_{\sigma}\right)$ ). Отметим (см, например, $[10$, гл. 7$])$, что для любого максимального $\sigma$-инвариантного тора $T$ справедливо равенство $G_{\sigma}=T_{\sigma} O^{p^{\prime}}\left(G_{\sigma}\right)$. (В [10] $G_{\sigma}$ обозначена через $\widehat{G}$.)

В дальнейшем нам потребуется следующая

Лемма 2.2. Пусть $R=S * M-\sigma$-инвариантная редуктивная подгруппа максимального ранга связной простой группы $G$, где $S$ - иентральный тор, а $M$ - полупростая подгруппа в $G$. Пусть простые компоненты группы $M_{\sigma}-$ это $G_{1}, \ldots, G_{k}$. Пусть $z_{i, j}=\left|Z\left(G_{i}\right) \cap Z\left(G_{j}\right)\right|, z_{i}=\left|Z\left(G_{i}\right) \cap S_{\sigma}\right|$. Тогда

$$
O^{p^{\prime}}\left(R_{\sigma}\right)=G_{1} * \cdots * G_{k} * S_{\sigma} \quad u \quad\left|R_{\sigma}: O^{p^{\prime}}\left(R_{\sigma}\right)\right| \leqslant \prod_{i \neq j} z_{i, j} \prod_{i} z_{i}
$$

ДокАЗАтЕЛЬСтво. Равенство $O^{p^{\prime}}\left(R_{\sigma}\right)=G_{1} * \cdots * G_{k} *\left(S_{\sigma}\right)$ хорошо известно, поэтому докажем лишь второе неравенство. В работе [3, предложение 2.4.2] доказано, что $\left|R_{\sigma}\right|=\left|M_{\sigma}\right| \cdot\left|S_{\sigma}\right|=\left|G_{1}\right| \cdots\left|G_{k}\right| \cdot\left|S_{\sigma}\right|$. Поскольку $G_{i} \cap G_{j}=Z\left(G_{i}\right) \cap Z\left(G_{j}\right)$ при $i \neq j$, $\left|G_{1} * \cdots * G_{k} *\left(S_{\sigma}\right)\right| \leqslant\left|R_{\sigma}\right| /\left(\prod_{i \neq j} z_{i, j} \prod_{i} z_{i}\right)$, откуда немедленно следует требуемое неравенство.

Далее мы докажем вспомогательный результат, которьй будет использоваться при изучении абелевых подгрупп в конечных группах Шевалле.

ЛЕмма 2.3. Пусть $G$ - связная редуктивная линейная алгебраическая группа над алгебраически замкнутым полем характеристики $p, R$ - ее редуктивная (не обязательно связная) подгруппа максимального ранга, причем $\left(\left|R: R^{0}\right|, p\right)=1$, $s \in R^{0}$ - некоторый полупростой әлемент; $T$ - произвольный максимальный тор 
в $R^{0}$, содержащий әлемент s. Тогда группа $C_{R}(s)$ редуктивна (хотя не обязательно связна). Она порождается тором $T$ вместе с теми корневыми подгруппами $U_{\alpha}$, для которых $\alpha(s)=1$, и теми представителями әлементов группь Вейля $n_{w} \in N_{R}(T)$, которые коммутируют $c s$. Компонента единиць $C_{R}(s)^{0}$ порождается тором $T$ и теми $U_{\alpha}$, для которых $\alpha(s)=1$. В частности, группа $C_{R}(s) / C_{R}(s)^{0}$ изоморфна некоторой секции группь Вейля для $G$. Более того, все унипотентные әлементы из $C_{R}(s)$ лежат в $C_{R}(s)^{0}$.

ДокАЗАТЕЛЬство. Зафиксируем подгруппу Бореля $B$ группы $R^{0}$, содержащую $T$. Ясно, что все указанные в леммепорождающие лежат в $C_{R}(s)$. Докажем, что $C_{R}(x)$ порождается указанньпи в лемме элементами. Покажем сначала, что в группе $R$ (которая не обязательно связна) имеет место разложение Брюа. Пусть $x$ - произвольньй элемент из $R$. Тогда $B^{x}$ - некоторая подгрупша Бореля групшы $R^{0}$. В силу леммы 2.1 сушествует такой элемент $s \in R^{0}$, что $B^{x}=B^{s}$. Тогда элемент $x s^{-1}$ нормализует подгрупу $B$. Тор $T^{x s^{-1}}$ является максимальньм тором групшы $B$. Поскольку все максимальные торы в $B$ сопряжены (лемма 2.1), существует такой элемент $g$ из $B$, что $T^{x s^{-1}}=T^{g}$. Поэтому можно считать, что $x s^{-1}$ нормализует тор $T$. Тогда $x s^{-1}=n_{w} t$ для некоторого $n_{w} \in N_{R}(T), t \in T$. Поскольку $t$ нормализует $B$ и $x s^{-1}$ нормализует $B$, элемент $n_{w}$ также нормализует $B$; значит, он нормализует $U$ - максимальную (связную) унипотентную подгруппу групшы $B$. Поскольку $s$ лежит в $R^{0}$, для него существует разложение Брюа, т.е. он представим в виде $u_{1} n_{w_{1}} t_{1} v_{1}$, где $u_{1} \in U \cap n_{w_{1}} U^{-} n_{w_{1}}^{-1}, n_{w_{1}} \in N_{R^{0}}(T), t \in T$ и $v_{1} \in U$. Поэтому элемент $x$ представим в виде $x=n_{w} t u_{1} n_{w_{1}} t_{1} v_{1}$. Поскольку элементы $t$ и $n_{w}$ нормализуют $U$, мы получаем представление элемента $x$ в виде $x=u_{2} n_{w_{2}} t_{2} v_{2}$, причем $u_{2} \in U \cap n_{w_{2}} U^{-} n_{w_{2}}^{-1}, n_{w_{2}} \in N_{R}(T), t_{2} \in T$ и $v_{2} \in U$. Поскольку это разложение Брюа совпадает с разложением Брюа элемента $x$ в группе $G$, такое разложение единственно.

Если $x \in C_{R}(s)$, то с помощью разложения Брюа можно записать $x=u n_{w} t v$, где $v \in U, t \in T, u \in U \cap n_{w} U^{-} n_{w}^{-1}$. Поскольку $s$ нормализует $U, N(T), U^{-}$и коммутирует с $x$, единственность разложения влечет, что каждьй из $u, n_{w}, v$ коммутирует с $s$. Более того, поскольку $s$ нормализует каждую корневую подгруппу $U_{\alpha}$, единственность разложения группы $U$ в произведение корневых подгрупп $U_{\alpha}(\alpha>0)$ влечет, что $\alpha(s)=1$, как только $u$ или $v$ содержит нетривиальный множитель из $U_{\alpha}$. Таким образом, $x$ лежит в группе, порожденной тором $T$ и теми $U_{\alpha}, n_{w}$, которые перестановочны с $s$.

Поскольку $T$ и все $U_{\alpha} \mathrm{c} \alpha(s)=1$ связны, то подгруппа $H$, порожденная ими, замкнута, связна и нормальна в $C_{R}(s)$. Так как группа Вейля конечна, $\left|G_{R}(s): H\right|<\infty$; значит, $H=C_{R}(s)^{0}$.

Так как корни группы $C_{R}(s)$ относительно тора $T$ возникают парами (т.е. если $\alpha(s)=1$, то и $-\alpha(s)=1)$, группа $C_{R}(s)$ редуктивна. Действительно, если $C_{R}(s)$ имеет нетривиальный унипотентный радикал $V$, то он нормализуется тором $T$, значит, содержит некоторую корневую подгруппу $U_{\alpha}$. Радикал $V$ нормализуется корневой группой $U_{-\alpha}$, что дает неунипотентньй элемент в $V$; получили противоречие.

Поскольку $\left(\left|R: R^{0}\right|, p\right)=1$, все унипотентные элементы из грушшы $R$ лежат в $R^{0}$, поэтому все унипотентные элементы из $C_{R}(s)$ лежат в $C_{R^{0}}(s)$. Тот факт, что в связной редуктивной группе $R^{0}$ любой унипотентньй элемент из $C_{R^{0}}(s)$ лежит в $C_{R^{0}}(s)^{0}$, хорошо известен (см., например, [12, п. 2.2]).

Пусть $x$ - полупростой элемент в связной редуктивной линейной алгебраической группе $G$. Тогда в силу леммы $2.3 C_{G}(x)^{0}$ - связная редуктивная подгруппа максималь- 
ного ранга и $\left[C_{G}(x)^{0}, C_{G}(x)^{0}\right]$ - полупростая група, корневая система которой является аддитивно замкнутой подсистемой корневой системы групшы $G$. Такие подгрупшы в дальнейшем будут назьваться подсистемными подгруппами. Поскольку в данной работе изучаются конечные группы, особый интерес представляют элементы простого порядка $r \neq p$. Оказьвается, что имеет место следующая лемма.

ЛЕмма 2.4 [13, п. 14.1]. Пусть $G$ - простая связная линейная алгебраическая зруппа над алгебраически замкнутым полем характеристики $p>0$ и әлемент $x \in G$ имеет простой порядок $r \neq p$. Пусть

$$
C^{\prime}=\left[C_{G}(x)^{0}, C_{G}(x)^{0}\right]
$$

- подсистемная подгруппа. Если $\Delta$ - диаграмма Дынкина корневой системы группь $C^{\prime}$, то справедливо одно из следующих утверждений:

1) диаграмма $\Delta$ получается удалением вериин из диаграммы Дынкина груп$n b l$;

2) диаграмма $\Delta$ получается из расширенной диаграммы Дынкина әруппь $G$ удалением одной вершины $r_{i}$, әде $r=c_{i}$ - коэффичиент при корне $r_{i} \boldsymbol{\theta}$ разложении корня $r_{0}$.

В частности, если $r$ не является плохим простым числом для группь $G$, то $\operatorname{dim}\left(Z\left(C_{G}(x)^{0}\right)\right) \geqslant 1$.

В конце данного раздела напомним алгоритм нахождения всех подсистем системы корней $\Phi$, принадлежащий Борелю и ди Зибенталю [14]. Рассматривается расширенная диаграмма Дынкина системы $\Phi$. Диаграммы всех возможных подсистем системы $\Phi$ получаются вычеркиванием некоторого количества вершин из расширенной диаграммы Дьнкина для $\Phi$.

\section{3. Общее строение абелевых подгрупп в связных редуктивных линейных алгебраических группах, конечных группах Шевалле и группах Вейля.}

ЛЕмма 3.1. Пусть $G$ - связная редуктивная линейная алгебраическая группа, $A$ - ее замкнутая абелева подгруппа. Тогда справедливы следуюшие утверждения:

1) зруппа $A$ представима в виде $A_{s} \times A_{u}-$ прямого произведения своей полупростой и унипотентной частей соответственно (см. [11, п. 15.5]);

2 ) существует такая редуктивная подгруппа $R$ максимального ранга групnы $G$, что

$$
A \leqslant R, \quad A_{u} \leqslant R^{0} \quad u \quad A_{s} \cap R^{0}=A_{s 0} \leqslant Z\left(R^{0}\right)
$$

3) если $W_{R}=N_{R}(T) / T, W_{R 0}=N_{R^{0}}(T) / T$ для некоторого максимального тора $T$ әруппь $R$, то группа $A_{s} / A_{s 0}$ изоморфно вкладывается в груп$n y W_{R} / W_{R 0}$.

Если $A$ - конечная группа, состоящая из әлементов, неподвижсных относительно некоторого автоморфизма Фробениуса $\sigma$ группы $G$, то $R$ б-инвариантна. 
ДокАЗАТЕЛЬСТВО. Пусть $s$ - некоторьй полупростой элемент из $A_{s}$. Положим $R=$ $C_{G}(s)$. Ясно, что $A \leqslant R$. В силу леммы $2.3 A_{u} \leqslant R^{0}$ и $R^{0}$ - связная редуктивная подгруппа максимального ранга группы $G$. Подгруппа $R^{0}$ нормальна в $R$, следовательно, любой элемент из $R$ нормализует $Z\left(R^{0}\right)$ и, значит, нормализует $Z\left(R^{0}\right)^{0}$. В силу леммы $2.3\left(\left|R: R^{0}\right|, p\right)=1$. Если существует такой полупростой элемент $s_{1} \in A_{s}$, что $s_{1} \in R^{0}$, но $s_{1} \notin Z\left(R^{0}\right)$, то рассмотрим $C_{R}\left(s_{1}\right)$. Ясно, что $A \leqslant C_{R}\left(s_{1}\right)$. В силу леммы $2.3 C_{R}\left(s_{1}\right)^{0}$ - связная редуктивная подгруппа максимального ранга группы $G$. Как и раньше, $A_{u} \leqslant C_{R}\left(s_{1}\right)^{0}$ и любой элемент из $C_{R}\left(s_{1}\right)$ нормализует $Z\left(C_{R}\left(s_{1}\right)^{0}\right)^{0}$. Заменяя группу $R$ на $C_{R}\left(s_{1}\right)$, получим редуктивную подгруппу максимального ранга группы $G$, содержащую группу $A$, меньшей размерности. Размерность действительно уменьшается, поскольку уменьшается размерность компоненты единицы. Указанньй вьше процесс конечен, поскольку на каждом шаге размерность уменьшается, а размерность групшы $G$ конечна. Отметим, что если $A$ - абелева подгруппа некоторой конечной групшы лиева типа, то $A$ состоит из неподвижных точек относительно некоторого автоморфизма $\Phi$ робениуса $\sigma$ и поэтому групшы, получаемые на каждом шаге указанного вьше процесса, $\sigma$-инвариантны. Следовательно, если $A \leqslant G_{\sigma}$, то группа $R$ является $\sigma$-инвариантной.

Далее,

$$
A_{s} / A_{s 0} \cong A_{s} R^{0} / R^{0} \leqslant R / R^{0}
$$

В силу леммы 2.3 любой элемент из $R$ можно представить в виде $n_{w} x$, где $x \in R^{0}$; следовательно, группа $R / R^{0}$ изоморфна групе

$$
N_{R}(T) / N_{R^{0}}(T) \cong W_{R} / W_{R 0}
$$

для любого максимального тора $T$ из $R^{0}$.

Отметим в качестве простого следствия леммы 3.1 , что если $\Phi_{R}=\Phi$, то $A_{s}=A_{s 0} \leqslant$ $Z(G)$. Действительно, редуктивная группа $R$, о которой говорится в лемме, в этом случае совпадает с $G$ (и совпадает с $R^{0}$ ), но $A_{s} \cap R^{0}=A_{s} \leqslant Z\left(R^{0}\right)$.

В лемме 3.1 возникают секции группы Вейля, поэтому, необходимо найти порядки больших абелевых подгрупп в группах Вейля для всех простых алгебраических групш. Кроме того, в дальнейшем часто будет возникать ситуация, когда некоторая полупростая абелева подгрупша является множеством неподвижных точек относительно автоморфизма Фробениуса некоторого тора $T$ размерности $n$. Для оценки порядков таких подгруп нам потребуется следующая

ЛЕмма 3.2. Пусть $S$ - б-инвариантный тор связной простой алгебраической группь $G$, и его размерность равна $n$, где $\sigma=q \sigma_{0}$ - некоторый автоморфизм Фробениуса. Пусть $S^{g}$ - некоторый сопряжсеный с ним б-инвариантный тор группь $G$. Пусть $X(S)$ - группа рациональных характеров тора $S$. Тогда существует такой әлемент $w$ из группы Вейля $W$ группь $G$, что $X(S)^{w} \subseteq X(S)$ и әруппа $\left(S^{g}\right)_{\sigma}$ изоморфна аруппе $X(S) /(\sigma w-1) X(S)$. В частности, поскольку әлемент $\sigma_{0} w$ имеет конечный порядок, $\left|\left(S^{g}\right)_{\sigma}\right| \leqslant(q+1)^{n}$.

ДокАЗАтЕльство. Поскольку тор $S \sigma$-инвариантен, его централизатор $C$ в группе $G$ - связная $\sigma$-инвариантная редуктивная подгруппа максимального ранга. Тогда группа $C^{g}$ также $\sigma$-инвариантна. Групшы $C$ и $C^{g}$ содержат некоторые $\sigma$-инвариантные максимальные торы $T$ и $T_{1}[15$, п. 10.10]; без ограничения общности можно считать, что они также сопряжены с помощью элемента $g$. Пусть $W_{1}-$ группа Вейля групшы $C$. В $[1$, 
следствие из предложения 2] утверждается, что тогда элемент $g^{\sigma} g^{-1}$ лежит в $N_{G}(T)$, а его образ относительно канонического гомоморфизма группы $N_{G}(T)$ на группу Вейля лежит в $N_{W}\left(W_{1}\right)$. Предложение 8 из работы [1] утверждает, что тогда $\left(T^{g}\right)_{\sigma} \cong$ $X(T) /(\sigma w-1) X(T)$. Поскольку $S \leqslant Z(C), S$ лежит в $T$. В силу взаимно однозначного соответствия между замкнутыми подгрупшами тора $T$ и подгруппами его групшы характеров $X(T)$ (см. [16, гл. III]) имеем $\left(S^{g}\right)_{\sigma} \cong X(S) /(\sigma w-1) X(S)$.

В таблище 1 ниже мы приведем порядки больших абелевых подгрупп в группах Вейля классических простых групп. Группа $W\left(A_{n}\right)$ изоморфна групе $S_{n+1}$, порядки и строение больших абелевых подгрупп в этой групе найдены в работе [17], и мы приведем их в таблице 1 без доказательства.

Группы $W\left(B_{n}\right)$ и $W\left(C_{n}\right)$ изоморфны, поэтому мы рассмотрим лишш группу $W\left(B_{n}\right)$. Если $\Phi$ - корневая система типа $B_{n}$ и $e_{1}, \ldots, e_{n}$ - ортонормированньй базис евклидова пространства, в котором лежит система $B_{n}$, то $\Phi$ можно записать в виде

$$
\left\{ \pm e_{i} \pm e_{j}, i \neq j, \pm e_{i} ; i, j=1, \ldots, n\right\} .
$$

Группа Вейля $W\left(B_{n}\right)$ действует точно на множестве $\left\{ \pm e_{1}, \ldots, \pm e_{n}\right\}$ из $2 n$ векторов. Пусть $A$ - некоторая абелева подгруппа группы $W\left(B_{n}\right)$; тогда $I_{1}, \ldots, I_{k}-$ все $A$-орбиты из $\left\{ \pm e_{1}, \ldots, \pm e_{n}\right\}$. Рассмотрим группу $G$ преобразований евклидова пространства, натянутого на векторы $e_{1}, \ldots, e_{n}$, относительно которой множество $\left\{ \pm e_{1}, \ldots, \pm e_{n}\right\}$ инвариантно. Ясно, что $W\left(B_{n}\right) \leqslant G$. Более того, эти групшы изоморфны, но нам не требуется изоморфность этих групп. Найдем $a(G)$ и докажем, что $a(G)=a\left(W\left(B_{n}\right)\right)$. Из доказательства теоремы 1.1 из [17] следует, что

$$
|A| \leqslant\left|I_{1}\right| \times \cdots \times\left|I_{k}\right| \text {. }
$$

Пусть $f(2 n)$ - порядок большой абелевой подгруппы в групе $G$.

Предположим, что среди множеств $I_{1}, \ldots, I_{k}$ имеются множества нечетного порядка. Без ограничения общности можно считать, что таким множеством является множество $I_{1}$ и базисньй вектор $e_{1}$ лежит в $I_{1}$. Тогда вектор $-e_{1}$ не лежит в множестве $I_{1}$. Действительно, предположим, что вектор - $e_{1}$ лежит в $I_{1}$. Тогда в группе $A$ существует элемент $\sigma$, переводящий $e_{1}$ в $-e_{1}$. Но тогда порядок элемента $\sigma$ четен, и можно считать, что $\sigma-2$-элемент. Кроме того, $\sigma$ не лежит в стабилизаторе орбиты $I_{1}$ в $A-\operatorname{St}_{A}\left(I_{1}\right)$. Значит, его образ относительно естественного гомоморфизма $\varphi: A \rightarrow A / \operatorname{St}_{A}\left(I_{1}\right)$ также имеет четный порядок. Но $\left|A / \mathrm{St}_{A}\left(I_{1}\right)\right|=\left|I_{1}\right|$ нечетен; противоречие. Таким образом, элемент $-e_{1}$ лежит в некотором другом множестве, без ограничения общности можно считать, что это множество $I_{2}$. Так как $G$ является группой линейных преобразований евклидова пространства, для любого $\sigma \in G$ справедливо $\sigma\left(-e_{1}\right)=-\sigma\left(e_{1}\right)$. Поскольку группа $A$ действует транзитивно на множествах $I_{1}, \ldots, I_{k}$, отсюда следует, что если $\sigma \in \operatorname{St}_{A}\left(I_{1}\right)$, то $\sigma \in \operatorname{St}_{A}\left(I_{1}\right) \cap \operatorname{St}_{A}\left(I_{2}\right)$ и для любого $v \in I_{1}$ вектор $-v$ лежит в $I_{2}$. Пусть $m=\left|I_{1}\right|=\left|I_{2}\right|$. Тогда в силу предыдуших рассуждений

$$
|A| \leqslant m \cdot f(2 n-2 m) .
$$

Рассмотрим группу $A_{1}$, которая на орбитах $I_{3}, \ldots, I_{k}$ действует так же, как група $A$, а множество $I_{1} \cup I_{2}$ относительно действия групшы $A_{1}$ распадается на орбиты из двух элементов - $\pm v$. По построению $A_{1} \leqslant G$ и группа $A_{1}$ абелева. Кроме того,

$$
|A|=m \cdot\left|\operatorname{St}_{A}\left(I_{1}\right)\right|<2^{m} \cdot\left|\operatorname{St}_{A}\left(I_{1}\right)\right|=\left|A_{1}\right| .
$$


Поэтому относительно действия группы $A \in A(G)$ множество $\left\{ \pm e_{1}, \ldots, \pm e_{n}\right\}$ распадается на орбиты четного порядка. С помощью индукции по $n$ покажем, что все орбиты имеют порядок 2 или 4 и, следовательно, $f(2 n) \leqslant 2^{n}$. Действительно, если порядок некоторой орбиты групшы $A$ больше либо равен 6 , то, как и в нечетном случае, можно построить абелеву подгруппу порядка $2^{3} \cdot 2^{n-3}$, откуда $|A| \leqslant 6 \cdot 2^{n-3}<2^{3} \cdot 2^{n-3}$. Поэтому

$$
f(2 n) \leqslant 2^{n} .
$$

С другой стороны, существует абелева подгруппа групшы $W\left(B_{n}\right)$ и, значит, группы $G$, переводящая $e_{i}$ в $\pm e_{i}$ для всех $i$. Ее порядок равен $2^{n}$, значит, $a\left(W\left(B_{n}\right)\right)=2^{n}$.

Заметим, что $W\left(D_{n}\right)$ изоморфно вкладывается в группу $W\left(B_{n}\right)$ как подгрупша индекса 2 , поэтому

$$
a\left(W\left(D_{n}\right)\right) \leqslant a\left(W\left(B_{n}\right)\right) .
$$

При четном $n$ эта оценка достигается, апри нечетном - нет. Для дальнейших рассуждений нам достаточно существования этой оценки сверху, хотя нетрудно найти и точную оценку.

ТАБлИЦА 1

\begin{tabular}{|c|c|}
\hline Тип групшы $G$ & Порядок $a(W)$ \\
\hline \hline & $3^{k}$, если $n=3 k-1$ \\
$A_{n}$ & $4 \cdot 3^{k-1}$, если $n=3 k$ \\
& $2 \cdot 3^{k}$, если $n=3 k+1$ \\
\hline$B_{n}, C_{n}, D_{n}$ & $\leqslant 2^{n}$ \\
\hline
\end{tabular}

Далее мы приведем известные оценки порядков больших унипотентных абелевых подгрупп.

Лемма $3.3[5]-[8]$. Пусть $q=p^{\alpha}$. Тогда

1) [5] $a_{u}\left(A_{n}(q)\right)=q^{\left[(n+1)^{2} / 4\right]}$;

2) [5], [6], [8] $a_{u}\left(C_{n}(q)\right)=q^{n(n+1) / 2}$;

3) [5], [7] $a_{u}\left(B_{n}(q)\right)=q^{n(n-1) / 2+1}$ npu $n \geqslant 4, p \neq 2$;

4) [5], [7] $a_{u}\left(B_{3}(q)\right)=q^{5}$ npu $p \neq 2$;

5) [5], [7] $a_{u}\left(D_{n}(q)\right)=q^{n(n-1) / 2}$ npu $n \geqslant 4$;

6) [7] $a_{u}\left({ }^{2} D_{n}\left(q^{2}\right)\right)=q^{(n-2)(n-1) / 2+2}$ npu $n \geqslant 5$;

7) $[7] a_{u}\left({ }^{2} D_{4}\left(q^{2}\right)\right)=q^{6}$;

8) [8] $a_{u}\left({ }^{2} A_{n}\left(q^{2}\right)\right)=q^{\left[(n+1)^{2} / 4\right]}$.

В указанных работах изучались абелевы унипотентные подгруппь данных групп. Кроме того, в этих же работах приводится строение больиих унипотентных абелевых подгрупп.

Лемма 3.4 [17, лемма 4.5]. Пусть $q$ - степень нечетного простого числа $p$. Тогда справедливы следующие оченки:

1) $q^{9} \leqslant a_{u}\left(F_{4}(q)\right) \leqslant q^{14}$

2) $q^{16} \leqslant a_{u}\left(E_{6}(q)\right) \leqslant q^{20}$

3) $q^{27} \leqslant a_{u}\left(E_{7}(q)\right) \leqslant q^{32}$

4) $q^{36} \leqslant a_{u}\left(E_{8}(q)\right) \leqslant q^{61}$

5) $q^{12} \leqslant a_{u}\left({ }^{2} E_{6}\left(q^{2}\right)\right) \leqslant q^{20}$. 
Доказательство леммы 3.4 в [17] для групп $E_{6}(q), E_{7}(q)$ и $E_{8}(q)$ не использует нечетности $q$, поэтому оценки, указанные для этих групा в лемме, справедливы в любой характеристике. Оценки для остальных исключительных групп (кроме ${ }^{2} B_{2}(q),{ }^{2} G_{2}(q)$ и $\left.{ }^{2} F_{4}(q)\right)$ приведены в следуюшей лемме.

ЛЕмма 3.5. Справедливы следующие утверждения:

1) $a_{u}\left({ }^{3} D_{4}\left(q^{3}\right)\right)=q^{5}$;

2) $q^{12} \leqslant a_{u}\left({ }^{2} E_{6}\left(q^{2}\right)\right) \leqslant q^{20}$

3) $a_{u}\left(G_{2}(q)\right)=q^{3}$, если q не делится на 3 ;

4) $a_{u}\left(G_{2}(q)\right)=q^{4}$, если $q$ делится на 3 ;

5) $q^{11} \leqslant a_{u}\left(F_{4}(q)\right) \leqslant q^{17}$, если q четно.

ДоказАтЕльство. Хорошо известно (см., например, [18]), что в группе $G_{2}(q)$ существует унипотентная абелева подгруппа порядка $q^{3}$, если $q$ не делится на 3 , и абелева подгрупша порядка $q^{4}$, если $q$ делится на 3 . В группе ${ }^{3} D_{4}\left(q^{3}\right)$ существует абелева унипотентная подгрупа порядка $q^{5}$, в групе ${ }^{2} E_{6}\left(q^{2}\right)$ существует абелева подгруппа порядка $q^{12}$, в группе $F_{4}(q)$ для четного $q$ существует абелева подгруппа порядка $q^{12}$, поэтому мы докажем во всех групшах лишь оценку сверху.

Рассмотрим сначала группу $G_{2}(q)$. Рассмотрим параболическую подгрупу $P$, фактор Леви которой порождается коротким фундаментальньм корнем $r_{1}$. Тогда $P=$ $H A_{1}(q) U_{r_{2}}$, где $U_{r_{2}}=\left\langle X_{r}: r \geqslant r_{2}\right\rangle$. Здесь под порядком понимается частичный порядок, заданньй правилом

$$
r=\alpha_{1} r_{1}+\alpha_{2} r_{2} \geqslant 0 \Longleftrightarrow \alpha_{1} \geqslant 0, \alpha_{2} \geqslant 0 .
$$

Заметим, что $\left[U_{r_{2}}, U_{r_{2}}\right]=X_{r_{0}}$, поэтому группа $U_{r_{2}} / X_{r_{0}}=\overline{U_{r_{2}}}$ абелева. Ее можно снабдить антисимметричной билинейной формой $<\cdot, \cdot>>$ по правилу

$$
[u, v]=x_{r_{0}}(<\bar{u}, \bar{v}>) \quad \text { для } u, v \in U_{r_{2}} .
$$

В $[10$, c. 211$]$ приведена таблица констант, участвующих в коммутаторных соотношениях Шевалле в группе $G_{2}(q)$, из которой следует, что данная форма невырождена, если $q$ не делится на 3 . Размерность пространства $\overline{U_{r_{2}}}$ равна 4 , векторы

$$
\overline{x_{r_{2}}(1)}=x_{1}, \overline{x_{r_{1}+r_{2}(1)}}=x_{2}, \overline{x_{2 r_{1}+r_{2}(1)}}=x_{3}, \overline{x_{3 r_{1}+r_{2}(1)}}=x_{4}
$$

образуют базу этого пространства. Таким образом, вся унипотентная подгруппа $U$ группы $G_{2}(q)$ представима в виде $X_{r_{1}} U_{r_{2}}$. Пусть $A$ - большая абелева подгруппа группы $U$. Ясно, что $X_{r_{0}}=Z(U) \leqslant A$. Предположим, что образ грушы $A$ относительно естественного гомоморфизма $\varphi: A \rightarrow A /\left(A \cap U_{r_{2}}\right)$ не равен единице. Пусть $x$ - некоторый элемент из $A$, образ которого $x^{\varphi}$ неединичен. Тогда можно определить действие элемента $x^{\varphi}$ на пространстве $\overline{U_{r_{2}}}$ сопряжением; кроме того, существует единственный элемент $x_{r_{1}}(t)$ такой, что $x^{\varphi}=x_{r_{1}}^{\varphi}(t)$. Поскольку групша $A$ абелева, пространство $A \cap U_{r_{2}} / X_{r_{0}}$ содержится в пространстве собственных векторов преобразования $x^{\varphi}$. Для любого нетривиального элемента $x^{\varphi}$ его матрица в базе $x_{1}, x_{2}, x_{3}, x_{4}$ имеет вид (структурные константы выбираются так же, как в [10])

$$
\left(\begin{array}{cccc}
1 & t & t^{2} & -t^{3} \\
0 & 1 & 2 t & 3 t^{2} \\
0 & 0 & 1 & -3 t \\
0 & 0 & 0 & 1
\end{array}\right) .
$$


Если $p>3$, топространство собственных векторов элемента $x^{\varphi}$ имеет размерность 1 . Таким образом,

$$
|A|=\left|A /\left(A \cap U_{r_{2}}\right)\right| \cdot\left|\left(A \cap U_{r_{2}}\right) / X_{r 0}\right| \cdot\left|X_{r 0}\right| \leqslant q^{3} .
$$

Если $A^{\varphi}=\{1\}$, то $A \leqslant U_{r_{2}}$. В этом случае группа $A / X_{r_{0}}$ - это изотропное подпространство пространства $\overline{U_{r_{2}}}$, следовательно, его размерность не превосходит 2 . Поэтому

$$
|A|=\left|A / X_{r_{0}}\right| \cdot\left|X_{r_{0}}\right| \leqslant q^{3}
$$

Если $q$ делится на 3 , то тогда антисимметричная форма, заданная на пространстве $\overline{U_{r_{2}}}$, вырождена. Пусть $L\left(x_{2}, x_{3}\right)$ - линейная оболочка векторов $x_{2}$ и $x_{3}$. Факторпространство $\overline{U_{r_{2}}} / L\left(x_{2}, x_{3}\right)$ обладает невырожденной антисимметричной формой, индуцированной формой $<\cdot, \cdot>$. Пусть $A$ - большая абелева подгруппа групшы $U$. Если в $A$ существует элемент $x$ такой, что $x^{\varphi} \neq 1$, то группа $\left(A \cap U_{r_{2}}\right) / X_{r_{0}}$ содержится в пространстве неподвижных точек преобразования $x^{\varphi}$. Из матрицы преобразования $x^{\varphi}$, полученной ранее, следует, что пространство неподвижных относительно $x^{\varphi}$ векторов имеет размерность не более 2 . Таким образом,

$$
|A|=\left|A /\left(A \cap U_{r_{2}}\right)\right| \cdot\left|\left(A \cap U_{r_{2}}\right) / X_{r_{0}}\right| \cdot\left|X_{r_{0}}\right| \leqslant q^{4} .
$$

Если $A \leqslant U_{r_{2}}$, то образ групшы $A$ относительно гомоморфизма

$$
A / X_{r_{0}} \rightarrow\left(A / X_{r_{0}}\right) /\left(A / X_{r_{0}} \cap L\left(x_{2}, x_{3}\right)\right)
$$

- изотропное пространство, следовательно, его размерность равна 1. Поэтому

$$
|A|=\left|\left(A / X_{r_{0}}\right) /\left(A / X_{r_{0}} \cap L\left(x_{2}, x_{3}\right)\right)\right| \cdot\left|L\left(x_{2}, x_{3}\right)\right| \cdot\left|X_{r_{0}}\right| \leqslant q^{4} .
$$

Пусть, наконец, $p=2$. В этом случае размерность подпространства собственных векторов относительно действия любого нетривиального элемента $x$ из $A \backslash\left(A \cap U_{r_{2}}\right)$ равняется 2. Антисимметричная билинейная форма, определенная ранее, на этом подпространстве невырождена, следовательно, образ абелевой группы $A \cap U_{r_{2}}$ относительно естественного гомоморфизма

$$
\varphi: A \cap U_{r_{2}} \rightarrow\left(A \cap U_{r_{2}}\right) / X_{r_{0}}
$$

- изотропное пространство, размерность которого равна 1. Таким образом,

$$
|A|=\left|A /\left(A \cap U_{r_{2}}\right)\right| \cdot\left|\left(A \cap U_{r_{2}}\right) / X_{r_{0}}\right| \cdot\left|X_{r_{0}}\right| \leqslant q^{3} .
$$

Группа $G_{2}(q)$ рассмотрена.

Рассмотрим группу ${ }^{3} D_{4}\left(q^{3}\right)$. Рассмотрим параболическую подгруппу $P$ группы $D_{4}\left(q^{3}\right)$, диаграмма Дынкина фактора Леви которой получается после удаления вершшны $r_{2}$ из диаграммы Дынкина групшы $D_{4}\left(q^{3}\right)$. Тогда подгрупша $P \tau$-инвариантна (здесь $\tau$ - скручивающий автоморфизм порядка 3 ). Ее подгруппа неподвижных точек имеет вид $L U_{1}$, где $L$ - фактор Леви, изоморфньй $A_{1}\left(q^{3}\right) H$. Группа $U_{1}$ обладает свойством $\left[U_{1}, U_{1}\right]=\left(X_{r_{0}}\right)_{\sigma}=X$. Поэтому група $U_{1} / X=\overline{U_{1}}$ абелева. Ее можно записать в виде 
$\overline{U_{2}} \oplus \overline{U_{3}}$, где $\overline{U_{2}}-$ векторное пространство размерности 2 над полем $G F(q)$, а $\overline{U_{3}}-$ векторное пространство размерности 2 над полем $G F\left(q^{3}\right)$. Как и в случае группы $G_{2}(q)$, каждое из этих пространств можно снабдить невырожденной антисимметричной билинейной формой $<\cdot, \cdot>$ по правилу

$$
[u, v]=x_{r_{0}}(<\bar{u}, \bar{v}>) .
$$

Эта форма будет невырожденной, поскольку в корневой системе типа $D_{4}$ все корни имеют одинаковую длину и, следовательно, все константы, участвующие в коммутаторных соотношениях Шевалле, по модулю равны 1 . Как и в случае группы $G_{2}(q)$, определяется действие групшы $L$ на $\overline{U_{1}}$. Тогда для любого нетривиального унипотентного элемента из $L$ его подгруппа неподвижных точек в $\overline{U_{1}}$ имеет порядок не более, чем $q$. Таким образом, неравенства, аналогичные неравенствам, записанным в случае группы $G_{2}(q)$, доказьвают утверждение леммы для групшы ${ }^{3} D_{4}\left(q^{3}\right)$.

Рассмотрим группу ${ }^{2} E_{6}\left(q^{2}\right)$. Рассмотрим в групе $E_{6}\left(q^{2}\right)$ параболическую подгруппу, фактор Леви которой изоморфен группе $A_{5}\left(q^{2}\right) H$. Тогда эта параболическая подгруппа $\tau$-инвариантна, где $\tau$ - скручивающий автоморфизм порядка 2 . Подгруппу ее неподвижных точек можно записать в виде $L U_{1}$, где $L$ - фактор Леви, изоморфньй группе ${ }^{2} A_{5}\left(q^{2}\right) H$, а $U_{1}$ - унипотентньй радикал, обладающий свойством

$$
\left[U_{1}, U_{1}\right]=\left(X_{r_{0}}\right)_{\tau}=X=Z\left(U_{1}\right)
$$

Вновь группа $U_{1} / X=\overline{U_{1}}$ представима в виде $\overline{U_{2}} \oplus \overline{U_{3}}$, где $\overline{U_{2}}-$ векторное пространство размерности 12 над полем $G F(q)$, а $\overline{U_{3}}$ - векторное пространство размерности 4 над полем $G F\left(q^{2}\right)$. Каждое из этих пространств можно снабдить невырожденной билинейной антисимметричной формой $<\cdot, \cdot>$, определенной правилом

$$
[u, v]=x_{r_{0}}(<\bar{u}, \bar{v}>)
$$

Тогда любая унипотентная абелева группа $A$ представима в виде $A_{1} A_{2}$, где $A_{2}=A \cap U_{1}$, $A_{1} \leqslant{ }^{2} A_{5}\left(q^{2}\right)$, причем образ группы $A_{2}$ относительно естественного гомоморфизма $A_{2} \rightarrow A_{2} / X$ - прямая сумма изотропных подпространств. Таким образом,

$$
|A| \leqslant\left|A_{1}\right| \cdot\left|A_{2}\right| \leqslant q^{9} \cdot q^{6} \cdot q^{4} \cdot q \leqslant q^{20} .
$$

Рассмотрим, наконец, группу $F_{4}(q)$, где $q$ четно. В ней существует параболическая подгруппа $P=L U_{r_{1}}$, диаграмма Дьнкина фактора Леви которой получается удалением длинного корня $r_{1}$. Тогда фактор Леви $L$ этой групшы изоморфен $C_{3}(q) H$. Группа $U_{r_{1}}$ обладает свойством $\left[U_{r_{1}}, U_{r_{1}}\right]=X_{r_{0}}$. Кроме того, $\mid Z\left(U_{r_{1}} \mid=q^{7}\right.$. Факторгруппа $U_{r_{1}} / Z\left(U_{r_{1}}\right)=\overline{U_{r_{1}}}$ элементарная абелева, и ее можно рассматривать как векторноепространство размерности 8 над полем $G F(q)$. На пространстве $\overline{U_{r_{1}}}$ вновь можно задать антисимметричную билинейную форму. Тогда если $A$ - некоторая унипотентная абелева подгруппа группы $F_{4}(q)$, то $A$ представима в виде $A_{1} A_{2}$, где $A_{1} \leqslant C_{3}(q)$, a $A_{2} \leqslant U_{r_{1}}$. Образ групшы $A_{2}$ относительно естественного гомоморфизма $A \rightarrow A /\left(A \cap Z\left(U_{r_{2}}\right)\right)$ изотропное подпространство; следовательно,

$$
|A| \leqslant\left|A_{1}\right| \cdot\left|A_{2}\right| \leqslant q^{6} \cdot q^{4} \cdot q^{7} \leqslant q^{17} .
$$


4. Большие абелевы подгруппы конечных групп лиева типа. В данном разделе результаты, изложенные ранее, будут применяться при изучении конечных групп лиева типа. Будет доказано, что большая абелева подгруппа в конечных простьх группах лиева типа, за исключением груп небольшого ранга, совпадает с большей унипотентной абелевой подгруппой. В непростых групах лиева типа большая абелева подгруппа равна произведению большой абелевой унипотентной подгруппы и центра соответствуюшей групш. Отметим, что большие абелевы подгрушы для групп $A_{n}(q)$ и $C_{l}(q), l \geqslant 3$, найдены в работе [17]. Группы ${ }^{2} B_{2}\left(2^{2 n+1}\right)$ и ${ }^{2} G_{2}\left(3^{2 n+1}\right)$ изучены в работах $[19]$ и [20]. Групшы ${ }^{2} F_{4}(q)$ в данной работе изучаться не будут.

Далее напомним строение $\sigma$-неподвижных точек редуктивных $\sigma$-инвариантных подгрупп максимального ранга в линейных простых алгебраических группах (см. [1, предложения $1,2,6$ и 8$]$ ).

ЛЕмма 4.1. Пусть $G$ - простая связная линейная алгебраическая группа; $\sigma$ ее автоморфизм Фробениуса; $G_{1}=M * S-\sigma$-инвариантная связная редуктивная подгруппа максимального ранга, где $M$ полупроста, а $S$ - чентральный тор; $G_{1}^{g}-$ некоторая сопряженная с ней б-инвариантная подгруппа. Пусть $\Delta_{1}-$ диаграмма Дынкина группь $G_{1} u W_{1}$ - группа Вейля группь $G_{1}$. Тогда

$$
g^{\sigma} g^{-1} \in N_{G}\left(G_{1}\right) \cap N_{G}(T)=N
$$

(для некоторого максимального тора $T$ из $G_{1}$ ) и существует биекиия

$$
\pi: N \rightarrow N_{W}\left(W_{1}\right) / W_{1} .
$$

Пусть $\pi\left(g^{\sigma} g^{-1}\right)=w ; \tau$ обозначает образ әлемента $w$ относительно естественного гомоморфизма $\varphi: N_{W}\left(W_{1}\right) \rightarrow \operatorname{Aut}_{W}\left(\Delta_{1}\right)$, а также графовый автоморфизм группь $M$, соответствующий данной симметрии $\tau$. Тогда $\left(M^{g}\right)_{\sigma} \cong M_{\sigma \tau}$.

Пусть $\overline{P_{1}}$ - подрешетка группы $X(T)$, порожденная всеми рациональными линейными комбинаииями корней из $\Delta_{1}$. Тогда

$$
\left(S^{g}\right)_{\sigma} \cong\left(X(T) / \overline{P_{1}}\right) /(\sigma w-1)\left(X(T) / \overline{P_{1}}\right)
$$

Kроме того, $\left|\left(G_{1}^{g}\right)_{\sigma}\right|=\left|M_{\sigma}^{g}\right| \cdot\left|S_{\sigma}^{g}\right|$.

Отметим, что в лемме 4.1 рассматривается група $G_{\sigma}$. В том случае, когда $G$ не является односвязной группой, конечная групша $O^{p^{\prime}}\left(G_{\sigma}\right)$ не совпадает с $G_{\sigma}$, поэтому порядок групшы $\left(G_{1}^{g}\right)_{\sigma} \cap O^{p^{\prime}}\left(G_{\sigma}\right)$ меньше, чем тот, что указан в лемме. В этом случае $G_{\sigma}=\widehat{H} O^{p^{\prime}}\left(G_{\sigma}\right)$, причем $|\widehat{H}: H|=d_{1} / d$. Здесь $\widehat{H}$ - максимальньй тор групшы $G_{\sigma} ; H-$ максимальньй тор группы $O^{p^{\prime}}\left(G_{\sigma}\right) ; d_{1}$ - порядок центра групшы $O^{p^{\prime}}\left(G_{\sigma}\right) ; d$ - порядок центра групшы $\left(G_{s c}\right)_{\sigma}$. Поэтому для того, чтобы получить порядок подгруппы $\left(G_{1}^{g}\right)_{\sigma}$ в группе $O^{p^{\prime}}\left(G_{\sigma}\right)$, порядок, указанньй в лемме, необходимо умножить на дробь $d_{1} / d$. Действительно, связная редуктивная подгруппа максимального ранга содержит некоторьй максимальньй тор связной простой линейной алгебраической, и поэтому

$$
\left(G_{1}^{g}\right)_{\sigma}=\widehat{H}\left(\left(G_{1}^{g}\right)_{\sigma} \cap O^{p^{\prime}}\left(G_{\sigma}\right)\right) .
$$

Значит,

$$
\left|\left(G_{1}^{g}\right)_{\sigma}:\left(\left(G_{1}^{g}\right)_{\sigma} \cap O^{p^{\prime}}\left(G_{\sigma}\right)\right)\right|=\frac{d_{1}}{d}
$$


следовательно,

$$
\left|\left(G_{1}^{g}\right)_{\sigma} \cap O^{p^{\prime}}\left(G_{\sigma}\right)\right|=\frac{d_{1}}{d}\left|\left(G_{1}^{g}\right)_{\sigma}\right| .
$$

Рассмотрим различные типы конечных груп Шевалле. Пусть далее мы находимся в обозначениях леммы 4.1. Пусть $G$ - простая связная линейная алгебраическая группа с системой корней $\Phi, \sigma$ - ее автоморфизм $\Phi$ робениуса $\left(\sigma=q \sigma_{0}, q=p^{\alpha}\right)$ и $G_{0}=O^{p^{\prime}}\left(G_{\sigma}\right)$. Пусть $A=A_{s} \times A_{u}$ - некоторая абелева подгрупша групшы $G_{0}$. Можно считать, что $A_{s} \nless Z\left(G_{0}\right)$. По лемме 3.1 в $G$ есть связная редуктивная $\sigma$-инвариантная подгруппа $R$ ( $R^{0}$ в обозначениях леммы 3.1) максимального ранга, содержащая группу $A_{0}=A_{u} \times A_{s 0}\left(A_{s 0}=A_{s} \cap R\right)$, причем группа $A / A_{0}$ изоморфна некоторой секции группы Вейля $W(\Phi), A_{s 0} \leqslant Z(R)$. Положим $S=Z(R)^{0}$.

Tй ${ }^{2} A_{n}$.

ЛЕмма 4.2 [2, предложение 8]. В обозначениях леммы 4.1 пусть $G$ - группа типа $A_{n}$ и пусть $G_{\sigma}$ - скрученный вид группы $G$. Пусть $G_{1}-\sigma$-инвариантная редуктивная подгруппа максимального ранга в группе $G$, соответствующая разбиению $\lambda$ числа $n+1$. Пусть $G_{1}^{g}-\sigma$-инвариантная редуктивная подгруппа группь $G$, полученная скручиванием группы $G_{1}$ әлементом $w \in W$, определенным правилом $\pi\left(g^{\sigma} g^{-1}\right)=w$. Предположим, что $w$ переходит в $\tau$ относительно гомоморфизма $N_{W}\left(W_{1}\right) \rightarrow \operatorname{Aut}_{W}\left(\Delta_{1}\right)$. Пусть $n_{i}-$ количество частей разбиения $\lambda$, равных $i$, так что $\operatorname{Aut}_{W}\left(\Delta_{1}\right) \cong S_{n_{2}} \times S_{n_{3}} \times \cdots$. Предположим, что $\sigma_{0} \tau\left(\left|\sigma_{0}\right|=2\right)$ дает разбиения $\mu^{(2)}, \mu^{(3)}, \ldots$ чисел $n_{2}, n_{3}, \ldots$ соответственно. Тогда простые компоненты полупростой әруппьь $\left(M^{g}\right)_{\sigma}$ имеют muп $A_{i-1}\left(q^{\mu_{j}^{(i)}}\right)$ для четных $\mu_{j}^{(i)}$ u mun ${ }^{2} A_{i-1}\left(q^{2 \mu_{j}^{(i)}}\right)$ для нечетного $\mu_{j}^{(i)}$.

Порядок полупростой части $\left(S^{g}\right)_{\sigma}$ әруппы $\left(G_{1}^{g}\right)_{\sigma}$ дается формулой

$$
(q+1)\left|\left(S^{g}\right)_{\sigma}\right|=\prod_{i, j: \mu_{j}^{(i)} \text { четно }}\left(q^{\mu_{j}^{(i)}}-1\right) \prod_{i, j: \mu_{j}^{(i)} \text { нечетно }}\left(q^{\mu_{j}^{(i)}}+1\right) .
$$

Поскольку группа $A / A_{0}$ изоморфна некоторой секции групшы Вейля $W\left(A_{n}\right) \cong S_{n+1}$, из таблишы 1 следует, что $\left|A / A_{0}\right| \leqslant 3^{(n+1) / 3}$. Поэтому, используя леммы 3.3 и 4.2 , можно записать следующую оценку:

$$
\begin{aligned}
(q+1)|A| \leqslant & \frac{d_{1}}{d} 3^{(n+1) / 3} \prod_{i, j: \mu_{j}^{(i)} \text { четно }}\left(q^{\mu_{j}^{(i)}}-1\right) \prod_{i, j: \mu_{j}^{(i)} \text { нечетно }}\left(q^{\mu_{j}^{(i)}}+1\right) \\
& \times \prod_{i, j}\left(i, q^{\left.\mu_{j}^{(i)}-1\right) q^{\mu_{j}^{(i)}\left[i^{2} / 4\right]} .}\right.
\end{aligned}
$$

Положим $S=Z(R)^{0}$. Поскольку для корневой системы типа $A_{n}$ нет плохих простых чисел, из леммы 2.4 следует, что $\operatorname{dim}(S) \geqslant 1$. Рассмотрим сначала случай, когда $\operatorname{dim}(S)=1$. Тогда группа $R$ имеет лишш две простые компоненты, поскольку в противном случае размерность центрального тора будет больше 1. Следовательно,

$$
R=A_{m_{1}-1}(K) * A_{m_{2}-1}(K) * S,
$$

причем $m_{1}+m_{2}=n+1$. Покажем, что в этом случае $R=C_{A_{n}(K)}(S)$. Действительно, централизатор тора в связной редуктивной групе связен, поэтому $R \leqslant C_{A_{n}(K)}(S)$. 
$\mathrm{C}$ другой стороны, единственная подсистемная связная подгруппа, содержащая $A_{n_{1}-1}(K) * A_{n_{2}-1}(K),-$ это $A_{n}(K)$. Поскольку ее центр имеет размерность 0 , он не совпадает с $S$. Таким образом, любой элемент из $A_{n}(K)$, централизующий $S$, лежит в $R$.

Предположим, что $A_{s} \neq A_{s 0}$. Поскольку группа $A_{s}$ нормализует $S$ и $A_{s 0} \in R$, мы получаем действие группы $A_{s} / A_{s 0}$ на групше характеров $X(S)$ тора $S$ (как группы автоморфизмов) по следующему правилу. Если $x \in A_{s} / A_{s 0}$ и $\chi \in X(S)$, то для любого $s \in S$ имеет место равенство $\chi^{x}(s)=\chi\left(s^{x}\right)$. Здесь под $s^{x}$ понимается сопряжение любым представителем элемента $x$ в группе $A_{s}$. Ясно, что это сопряжение не зависит от выбора представителя, поскольку $A_{s 0}$ централизует $S$. Действие элемента $x$ является автоморфизмом группы $X(S)$. Действительно, сохранение операции очевидно, так что $x$ является гомоморфизмом группы $X(S)$. Поскольку у каждого элемента в групе существует обратньй, действие элемента $x$ биективно, значит, является автоморфизмом группы $X(S)$. Поскольку размерность тора $S$ равна единице, группа $X(S)$ изоморфна группе $\mathbb{Z}$. Единственньй нетривиальный автоморфизм групшы $\mathbb{Z}$ - это автоморфизм порядка 2. Таким образом, если группа $A_{s} / A_{s 0}$ нетривиальна, то ее порядок равен 2.

Кроме того, в этом случае $\left|A_{s 0} \cap S\right| \leqslant 2$. Действительно, $A_{s}$ - абелева группа, поэтому любой элемент из $A_{s 0}$ неподвижен относительно действия элемента $x$. В силу определения действия элемента $x$ на $X(S)$ отсюда следует, что для порождающего характера $\chi$ группы $X(S)$ и любого $t \in A_{s 0} \cap S$ выполняется равенство

$$
\chi(t)=\chi^{-1}\left(t^{x}\right)=\chi^{-1}(t)=\chi\left(t^{-1}\right),
$$

т.е. $t^{-1}=t$, в частности, $\left|A_{s 0} \cap S\right| \leqslant 2$. Поскольку $d_{1} / d \leqslant 1$, множитель $d_{1} / d$ можно заменить на единицу. Множитель $3^{(n+1) / 3}$ возникал при оценке порядка групшы $A / A_{0}$. Поскольку в нашем случае $\left|A / A_{0}\right|=2$, множитель $3^{(n+1) / 3}$ заменяется на 2 . Кроме того, поскольку $\left|S \cap A_{0}\right| \leqslant 2$, вместо $|S|$, участвующего в неравенстве (1), также можно оставить 2. Таким образом, ввиду (1)

$$
|A| \leqslant 4\left(m_{1}, q+1\right) q^{\left[m_{1}^{2} / 4\right]} \cdot\left(m_{2}, q+1\right) q^{\left[m_{2}^{2} / 4\right]},
$$

либо

$$
|A| \leqslant 4\left(\frac{n+1}{2}-1, q^{2}-1\right) q^{2[(n+1) / 16]}
$$

(вторая возможность возникает, когда $m_{1}=m_{2}$, т.е. $n+1$ четно). Покажем, что при $n \neq 2$ и $q \neq 3$ в обоих случаях $|A| \leqslant q^{\left[(n+1)^{2} / 4\right]}$.

При $n \geqslant 14$ имеем

$$
\begin{aligned}
4\left(m_{1}, q+1\right) q^{\left[m_{1}^{2} / 4\right]} \cdot\left(m_{2}, q+1\right) q^{\left[m_{2}^{2} / 4\right]} & \leqslant 4(q+1)^{2} \cdot q^{m_{1}^{2} / 4+m_{2}^{2} / 4} \leqslant q^{6} \cdot q^{m_{1}^{2} / 4+m_{2}^{2} / 4} \\
& \leqslant q^{m_{1}^{2} / 4+m_{2}^{2} / 4+7-1} \leqslant q^{m_{1}^{2} / 4+m_{2}^{2} / 4+n / 2-1} \\
& \leqslant q^{m_{1}^{2} / 4+m_{2}^{2} / 4+m_{1} m_{2} / 2-1} \leqslant q^{\left[(n+1)^{2} / 4\right]} .
\end{aligned}
$$

Если $8 \leqslant n \leqslant 13, m_{1}, m_{2}>1$, то

$$
\begin{aligned}
4\left(m_{1}, q+1\right) q^{\left[m_{1}^{2} / 4\right]} \cdot\left(m_{2}, q+1\right) q^{\left[m_{2}^{2} / 4\right]} & \leqslant 4(q+1)^{2} \cdot q^{m_{1}^{2} / 4+m_{2}^{2} / 4} \leqslant q^{6} \cdot q^{m_{1}^{2} / 4+m_{2}^{2} / 4} \\
& \leqslant q^{m_{1}^{2} / 4+m_{2}^{2} / 4+8-2} \leqslant q^{m_{1}^{2} / 4+m_{2}^{2} / 4+n-2} \\
& \leqslant q^{m_{1}^{2} / 4+m_{2}^{2} / 4+m_{1} m_{2} / 2-1} \leqslant q^{\left[(n+1)^{2} / 4\right]} .
\end{aligned}
$$


Если $6 \leqslant n \leqslant 7, q>2$ и $m_{1}, m_{2}>1$, то

$$
\begin{aligned}
4\left(m_{1}, q+1\right) q^{\left[m_{1}^{2} / 4\right]} \cdot\left(m_{2}, q+1\right) q^{\left[m_{2}^{2} / 4\right]} & \leqslant 4(q+1)^{2} \cdot q^{m_{1}^{2} / 4+m_{2}^{2} / 4} \leqslant q^{4} \cdot q^{m_{1}^{2} / 4+m_{2}^{2} / 4} \\
& \leqslant q^{m_{1}^{2} / 4+m_{2}^{2} / 4+6-2} \leqslant q^{m_{1}^{2} / 4+m_{2}^{2} / 4+n-2} \\
& \leqslant q^{m_{1}^{2} / 4+m_{2}^{2} / 4+m_{1} m_{2} / 2-1} \leqslant q^{\left[(n+1)^{2} / 4\right]} .
\end{aligned}
$$

Если $8 \leqslant n \leqslant 13, q>2$ и $m_{1}=1$, то

$$
4(n, q+1) q^{\left[n^{2} / 4\right]} \leqslant 4(q+1) \cdot q^{n^{2} / 4} \leqslant q^{3} \cdot q^{n^{2} / 4} \leqslant q^{n^{2} / 4+4-1} \leqslant q^{\left[(n+1)^{2} / 4\right]} .
$$

Если $n=7, q>2$ и $m_{1}=1$, то $4(7, q+1) q^{12}<q^{16}$. Если $n=6, q>2$ и $m_{1}=1$, то $4(6, q+1) q^{9}<q^{12}$. Если $n=5, q>2$ и $m_{1}=1$, то $4(5, q+1) q^{6}<q^{9}$. Если $n=5$, $q>2, m_{1}=2, m_{2}=4$, то $4(2, q+1) q(4, q+1) q^{4}<q^{9}$. Если $n=5, q>2, m_{1}=3$, $m_{2}=3$, то $4(3, q+1) q^{2}(3, q+1) q^{2}<q^{9}$. Аналогичньм образом разбираются оставшиеся случаи. Далеев подобных ситуациях мы будем опускать вычисления и записьвать лишг результат.

Рассмотрим отдельно группу ${ }^{2} A_{2}\left(3^{2}\right)$, при этом $m_{1}=1, m_{2}=2$. Порядок ее большой абелевой подгруппы равен 16 (это некоторьй максимальный тор, который изоморфен $\mathbb{Z}_{4} \times \mathbb{Z}_{4}$ и единственен с точностью до сопряжения). Она содержится в групше $A_{2}(K)$, где $K$ - алгебраически замкнутое поле характеристики 3 , причем $A_{2}(K)$ односвязна, т.е. $\Gamma_{\pi}=\Gamma_{s c}$. Группа $R=M * S$, где $M$ - полупростая группа типа $A_{1}, S$ - тор размерности 1 и $M \cap S$ конечна. Если $M$ - группа присоединенного типа, то ее центр тривиален и тогда $|A| \leqslant|S| \cdot\left|a\left(A_{1}(3)\right)\right| \leqslant 4 \cdot 3<16$. Если $M$ односвязна, то ее центр нетривиален и его порядок равен 2. Покажем, что в этом случае пересечение $M \cap S$ совпадает с центром группы $M$. Действительно, $M=\left\langle X_{r}, X_{-r}\right\rangle, r$ - некоторьй корень корневой системы типа $A_{2}$. Пусть $P$ - решетка, порожденная корнем $r, \bar{P}$ - подрешетка решетки $\Gamma_{s c}$, порожденная всеми возможными рациональньми комбинациями корня $r$. Тогда $S=\bar{P}^{\perp}$. В алгебраической групе типа $A_{2}\left|\Gamma_{s c}: \Gamma_{a d}\right|=3$. Група $\bar{P}$ циклическая, пусть $\chi$ - ее порождающий элемент. Тогда либо $\chi \in P$, либо $\chi^{3} \in P$. Пусть $x$ - нетривиальньй элемент из центра группы $M$. Поскольку $|Z(M)|=2, x^{2}=1$. Тогда $x^{r}=1$, значит,

$$
1=x^{\chi^{3}}=x^{\chi^{2}} x^{\chi}=\left(x^{2}\right)^{\chi} x^{\chi}=x^{\chi}=1,
$$

поэтому $x \in S$. Следовательно, и в этом случае $|A| \leqslant 4 \cdot 3<16$.

Пусть теперь $A_{s}=A_{s 0}$. В силу леммы $3.1 A_{s 0}$ содержится в центре группы $R$, поэтому порядок группы $A$ оценивается следующим образом:

$$
|A| \leqslant(q+1)\left(m_{1}, q+1\right) q^{\left[m_{1}^{2} / 4\right]}\left(m_{2}, q+1\right) q^{\left[m_{2}^{2} / 4\right]},
$$

либо

$$
|A| \leqslant(q-1)\left(\frac{n+1}{2}, q^{2}-1\right) q^{2\left[(n+1)^{2} / 16\right]} .
$$

Справедливо неравенство $|A| \leqslant q^{\left[(n+1)^{2} / 4\right]}$, когда $n \geqslant 5$. Случаи $n=2, n=3$ нужно разбирать отдельно, а при $n=4$ возможны следующие исключения: $m_{1}=1, m_{2}=4$, $q=3$ и $m_{1}=2, m_{2}=3, q=2$.

Рассмотрим случай $n=2$. Если $(3, q+1)=3$, то либо центр групшы ${ }^{2} A_{2}\left(q^{2}\right)$ нетривиален и тогда

$$
|A| \leqslant(q+1)\left(m_{1}, q+1\right) q^{\left[m_{1}^{2} / 4\right]}\left(n_{2}, q+1\right) q^{\left[m_{2}^{2} / 4\right]} \leqslant 3 q^{2},
$$


либо

$$
|A| \leqslant \frac{1}{3}(q+1)\left(m_{1}, q+1\right) q^{\left[m_{1}^{2} / 4\right]}\left(m_{2}, q+1\right) q^{\left[m_{2}^{2} / 4\right]} \leqslant q^{2} .
$$

Если $(3, q+1)=1$, то группа ${ }^{2} A_{2}\left(q^{2}\right)$ односвязна, но ее центр тривиален. Большая абелева подгруппа в этом случае - это максимальный тор порядка $(q+1)^{2}$, изоморфньй $\mathbb{Z}_{q+1} \times \mathbb{Z}_{q+1}$, которьй единственен с точностью до сопряжения. Вновь нужно разобрать случай, когда $m_{1}=1, m_{2}=2$. Как в случаегруппы ${ }^{2} A_{2}\left(3^{2}\right), M \cap S$ совпадает с центром группы $M$, поэтому

$$
|A| \leqslant(q+1) q<(q+1)^{2},
$$

где $(q+1)^{2}$ - порядок большой абелевой подгрупшы.

Пусть теперь $n=3$. Если $n_{1}=n_{2}=2$, то

$$
|A| \leqslant(q+1)(2, q+1)(2, q+1) q^{2} \leqslant q^{4}
$$

при $q \neq 3$. В группе ${ }^{2} A_{3}\left(3^{2}\right)$ имеются три возможности для центра. Центр может быть тривиален и тогда ${ }^{2} A_{3}\left(3^{2}\right)$ имеет присоединенный тип. Поэтому

$$
|A| \leqslant \frac{1}{4} \cdot 4 \cdot 2 \cdot 2 \cdot 3^{2} \leqslant 3^{4} .
$$

Центр может быть порядка 2 и тогда

$$
|A| \leqslant \frac{1}{2} \cdot 4 \cdot 2 \cdot 2 \cdot 3^{2} \leqslant 2 \cdot 3^{4} .
$$

И, наконец, центр может быть порядка 4 и тогда

$$
|A| \leqslant 4 \cdot 2 \cdot 2 \cdot 3^{2} \leqslant 4 \cdot 3^{4} .
$$

Если $m_{1}=1, m_{2}=3$, то как в случае ${ }^{2} A_{2}\left(3^{2}\right), M \cap S$ совпадает с центром группы $M$, поэтому $|A| \leqslant(q+1) q^{2} \leqslant q^{4}$.

Последние два случая ${ }^{2} A_{4}\left(2^{2}\right)$ и ${ }^{2} A_{4}\left(3^{2}\right)$ рассматриваются аналогично. Таким образом, случай, ког да размерность центрального тора равна 1 , разобран.

Пусть теперь размерность тора $S$ больше 1 . Для всех $m$ и $k$ справедливо неравенство

$$
\left(q^{k}+(-1)^{k+1}\right)\left(m, q^{k}+(-1)^{k+1}\right) q^{k\left[m^{2} / 4\right]} \leqslant(q+1)(m k, q+1) q^{\left[m^{2} k^{2} / 4\right]} .
$$

Кроме того, при $1 \leqslant m_{1} \leqslant m_{2}$, кроме случаев $m_{1}=1, m_{2}=1 ; m_{1}=1, m_{2}=2$ и $m_{1}=1, m_{2}=3, q=2$, справедливо неравенство

$$
(q+1)\left(m_{1}, q+1\right) q^{\left[m_{1}^{2} / 4\right]}(q+1)\left(m_{2}, q+1\right) q^{\left[m_{2}^{2} / 4\right]} \leqslant(q+1)\left(m_{1}+m_{2}, q+1\right) q^{\left[\left(m_{1}+m_{2}\right)^{2} / 4\right]} .
$$

Используя неравенства (2) и (3), выражение (1) приводится к одному из следующих видов:

$$
\begin{gathered}
(q+1)^{2} 3^{(n+1) / 3}\left(m_{1}, q+1\right) q^{\left[m_{1}^{2} / 4\right]}\left(m_{2}, q+1\right) q^{\left[m_{2}^{2} / 4\right]}\left(m_{3}, q+1\right) q^{\left[m_{3}^{2} / 4\right]} \\
\left(m_{1}+m_{2}+m_{3}=n+1\right) \\
(q+1)^{n-1} 3^{(n+1) / 3}(2, q+1) q \\
(q+1)^{n-3} 3^{(n+1) / 3}(2, q+1)^{2} q^{2} \\
3^{n+(n+1) / 3} 2^{2} \\
3^{n+(n+1) / 3-2} 2^{4}
\end{gathered}
$$


Последние два случая возможны лишь при $q=2$. Выражения (4)-(8) не превосходят порядка большой абелевой подгруппы при $n \geqslant 5$. Таким образом, необходимо рассмотреть лишь группы ${ }^{2} A_{n}\left(q^{2}\right)$ при $n \leqslant 4$ (при $n=4-$ лишь группу $\left.{ }^{2} A_{4}\left(2^{2}\right)\right)$. Все случаи разбираются однотипно, поэтому мы рассмотрим лишш случай $n=3$. При $n=3$ для связной редуктивной групшы $R$ существуют две различных возможности. Группа $R$ либо является максимальным тором, либо равна центральному произведению тора размерности 2 и группы типа $A_{1}$.

Пусть группа $R$ является максимальньп тором, т.е. $A=A_{s}$. Тогда $R$ - это гомоморфный образ групшы диагональных матрищ относительно естественного гомоморфизма $S L_{4}(K) \rightarrow S L_{4}(K) / Z$, где $Z \leqslant Z\left(S L_{4}(K)\right)$. Поскольку группа $A_{s}$ нормализует $R$, определим действие группы $A_{s} / A_{s 0}$ на $R$ правилом $s^{\bar{x}}=s^{x}$, где $s \in R, x \in A_{s}$ и $\bar{x} \in A_{s} / A_{s 0}$. Элементы из $A_{s} / A_{s 0}$ переставляют диагональные элементы грушшы $R$. Относительно действия группы $A_{s} / A_{s 0}$ множество $\{1,2,3,4\}$ (множество диагональных мест) разбивается на орбиты. Поскольку группа $A_{s}$ абелева, элементы из $A_{s} / A_{s 0}$ централизуют элементы из $A_{s 0}$, следовательно, элементы, стоящие на местах, соответствующих одной орбите, могут отличаться лишњ на множитель из $Z$. Значит, в группе $A_{s 0}$ существует подгруппа $A_{s 1}$ индекса не более чем $|Z|($ a $|Z| \leqslant 4)$, содержащаяся в торе, размерность которого на единицу меньше количества орбит грушшы $A_{s} / A_{s 0}$. Если существуют четыре орбиты, то $A_{s}=A_{s 0}$ и в силу леммы $3.2|A|$ не превосходит $(q+1)^{3}$, что не превосходит порядка большой абелевой подгрупшы, указанного в таблице 2. Если существуют три орбиты, то тогда порядок группы $A_{s} / A_{s 0}$ равен 2 , $\left|A_{s 0} / A_{s_{1}}\right| \leqslant(4, q+1),\left|A_{s 1}\right| \leqslant(q+1)^{2}$, т.е. $\left|A_{s}\right| \leqslant 2(4, q+1)(q+1)^{2}$, что вновь не превосходит порядка большой абелевой подгруппы, указанного в таблице 2 . Если существуют две орбиты, то $\left|A_{s} / A_{s 0}\right| \leqslant 4,\left|A_{s 0} / A_{s 1}\right| \leqslant(4, q+1),\left|A_{s 1}\right| \leqslant q+1$, откуда следует, что $\left|A_{s}\right| \leqslant 4(4, q+1)(q+1)$, т.е. порядок групшы $A_{s}$ вновь не превосходит порядка большой абелевой подгруппы. Если, наконец, существует лиш одна орбита, то $\left|A_{s} / A_{s 0}\right| \leqslant 4, A_{s 0} / A_{s 1}=A_{s 0} \leqslant Z$ и, значит, $\left|A_{s}\right| \leqslant 4(4, q+1)$.

Пусть теперь $R$ - это центральноепроизведение тора $S$ размерности 2 и простой связной группы $M$ типа $A_{1}$. В силу леммы $2.3 A_{s} \leqslant N(R)$. Из [2, лемма 1] следует, что $N(R) / R \cong K \ltimes\left(W_{R}^{\perp}\right)$, где $K$ изоморфна группе симметрий диаграммы Дьнкина полупростой части группы $R$, а $W_{R}^{\perp}=\left\langle w_{s}\right|(s, r)=0$ для всех $\left.r \in \Phi(R)\right\rangle$. Так как ранг $M$ равен 1 , группа $K$ тривиальна, а $W_{R}^{\perp} \leqslant S_{3}$. Следовательно, $\left|A_{s} / A_{s 0}\right| \leqslant 3$. Как и при доказательстве леммы 2.3 , в $R$ можно найти максимальньй тор $T$, которьй нормализует групп $A_{s}$. Тор $S$ содержится во всех максимальных торах, следовательно, он содержится в $T$. Как в предыдущем случае, определим действие группы $A_{s}$ на $T$. В некоторой базе пространства $K^{4}$ тор $T$ диагонализируем, и группа $A_{s} / A_{s 0}$ переставляет элементы, стоящие по диагонали. Если она нетривиальна, то в группе $A_{s 0}$ существует подгруппа индекса не более, чем порядок групшы $Z$, которая лежит в торе размерности не более 1 . Поэтому $|A|=\left|A_{s}\right| \cdot\left|A_{u}\right| \leqslant 4(4, q+1)(q+1) q$, что не превосходит порядка большой абелевой подгруппы, указанного в таблище 2 . Если $A_{s}$ совпадает с $A_{s 0}$, то $|A| \leqslant(q+1)^{2} q$, что вновь не превосходит порядка большой абелевой подгруппы.

Tип $D_{l}$. Применение леммы 4.1 в случае алгебраической групшы типа $D_{l}$ дает следующий результат.

ЛЕмма 4.3 [2, предложение 10]. Пусть в обозначениях леммы $4.1 \quad G$ - группа типа $D_{l}$ над алгебраически замкнутым полем характеристики $p$ и $G_{1}-\sigma$-инвариантная редуктивная подгруппа максимального ранга в $G$, определенная парой разбиений $\lambda, \mu$ с условием $|\lambda|+|\mu|=l$. Пусть $k_{i}$ - количество частей разбиения $\lambda$, 
равных $i$, и $n_{i}$ - количество частей разбиения $\mu$, равных $i\left(n_{1}=0\right)$. Тогда

$$
\operatorname{Aut}_{W}\left(\Delta_{1}\right) \cong\left\{\begin{array}{l}
S_{k_{2}} \times\left(\mathbb{Z}_{2} \nmid S_{k_{3}}\right) \times\left(\mathbb{Z}_{2} \nmid S_{k_{4}}\right) \times \cdots \times\left(\mathbb{Z}_{2} \nmid S_{n_{2}}\right) \times\left(\mathbb{Z}_{2} \nmid S_{n_{3}}\right) \times \cdots, \\
\quad \text { если } k_{1}>0, \\
\text { подгруппе индекса } 2 \text { в ней, если } k_{1}=0 .
\end{array}\right.
$$

Пусть $G_{1}^{g}-\sigma$-инвариантная подгруппа группь $G$, полученная скручиванием груп$n$ вы $G_{1}$ әлементом $w \in W$, определенного правилом $\pi\left(g^{\sigma} g^{-1}\right)=w$. Предполохим, что $w$ переходит в $\tau \in$ Aut $_{W}\left(\Delta_{1}\right)$. Предполохим, что $\sigma_{0} \tau\left(\sigma=q \sigma_{0}\right)$ дает пары разбиений $\xi^{(i)}, \eta^{(i)}$ с условием $\left|\xi^{(i)}\right|+\left|\eta^{(i)}\right|=k_{i}$ (әде $\eta^{(i)}$ nусто, если $\left.i=2\right)$ и пару разбиений $\zeta^{(i)}, \omega^{(i)}$ с условием $\left|\zeta^{(i)}\right|+\left|\omega^{(i)}\right|=n_{i}$. Тогда простые компоненты полупростой группь $\left(M^{g}\right)_{\sigma}$ имеют тип

$$
A_{i-1}\left(q^{\xi_{j}^{(i)}}\right), \quad{ }^{2} A_{i-1}\left(q^{2 \eta_{j}^{(i)}}\right), \quad D_{i}\left(q^{\zeta_{j}^{(i)}}\right), \quad{ }^{2} D_{i}\left(q^{2 \omega_{j}^{(i)}}\right)
$$

по одной компоненте для каждой части кажсдого из разбиений $\xi^{(i)}, \eta^{(i)} u \zeta^{(i)}, \omega^{(i)}$.

Eсли $k_{1}=0$, то общее количество компонент типа ${ }^{2} A_{i-1}$ с нечетным $i$ и типа ${ }^{2} D_{i}$ четно, если $\sigma_{0}=1$, и нечетно, если $\sigma_{0} \neq 1$.

Порядок тора $\left(S^{g}\right)_{\sigma}$ дается формулой

$$
\left|\left(S^{g}\right)_{\sigma}\right|=\prod_{i, j}\left(q^{\xi_{j}^{(i)}}-1\right) \prod_{i, j}\left(q^{\eta_{j}^{(i)}}+1\right) .
$$

Ввиду таблицы 1 и лемм 3.3, 4.3 справедливо следующее неравенство:

$$
\begin{aligned}
|A| \leqslant & 2^{l} \prod_{i, j}\left(q^{\xi_{j}^{(i)}}-1\right) \prod_{i, j}\left(q^{\eta_{j}^{(i)}}+1\right) \prod_{i, j}\left(i, q^{\xi_{j}^{(i)}}-1\right) q^{\xi_{j}^{(i)}\left[i^{2} / 4\right]} \prod_{i, j}\left(i, q^{\eta_{j}^{(i)}}+1\right) q^{\eta_{j}^{(i)}\left[i^{2} / 4\right]} \\
& \times \prod_{i, j}\left(4, q^{\zeta_{j}^{(i)}}-1\right) q^{\zeta_{j}^{(i)} \frac{i(i-1)}{2}} \prod_{j}\left(4, q^{\omega_{j}^{(4)}}+1\right) q^{6 \omega_{j}^{(4)}} \prod_{i>5, j}\left(4, q^{\omega_{j}^{(i)}}+1\right) q^{\omega_{j}^{(i)} \frac{(i-1)(i-2)+2}{2}} .
\end{aligned}
$$

Для любого $m \geqslant 3$ справедливо неравенство

$$
\left(q^{k}-1\right)\left(\frac{m}{k}, q^{k}-1\right) q^{k\left[m^{2} / 4 k^{2}\right]} \leqslant q^{m(m-1) / 2},
$$

и для любого $m \geqslant 4$ справедливо неравенство

$$
\left(q^{k}+1\right)\left(\frac{m}{k}, q^{k}+1\right) q^{k\left[m^{2} / 4 k^{2}\right]} \leqslant q^{m(m-1) / 2} .
$$

Далее, для любого $m$ выполняются неравенства

$$
\begin{gathered}
\left(4, q^{k}-1\right) q^{k \frac{(m / k)(m / k-1)}{2}} \leqslant q^{m(m-1) / 2} \\
\left(4, q^{k}+1\right) q^{k \frac{(m / k-1)(m / k-2)+2}{2}} \leqslant q^{((m-1)(m-2)+2) / 2} .
\end{gathered}
$$


Кроме того, для любых $m_{1}, m_{2}$ выполняются неравенства

$$
\begin{aligned}
(4, q-1) q^{m_{1}\left(m_{1}-1\right) / 2} q^{m_{2}\left(m_{2}-1\right) / 2} & \leqslant q^{\left(m_{1}+m_{2}\right)\left(m_{1}+m_{2}-1\right) / 2} \\
(4, q+1) q^{\left(\left(m_{1}-1\right)\left(m_{1}-2\right)+2\right) / 2} q^{\left(\left(m_{2}-1\right)\left(m_{2}-2\right)+2\right) / 2} & \leqslant q^{\left(\left(m_{1}+n_{2}-1\right)\left(m_{1}+m_{2}-2\right)+2\right) / 2} \\
(4, q+1) q^{6} \cdot q^{((m-1)(m-2)+2) / 2} & \leqslant q^{((m+4-1)(m+4-2)+2) / 2}
\end{aligned}
$$

Пусть $\sigma_{0}=1$, т.е. $O^{p^{\prime}}\left(\left(D_{l}(K)\right)_{\sigma}\right)$ - группа нормального типа. Тогда с помощью неравенств (10)-(16) правая часть выражения (9) приводится к виду

$$
\begin{aligned}
& 2^{l}(2(q-1) q)^{m_{1}}(2(q+1) q)^{m_{2}}\left((3, q+1)(q+1) q^{2}\right)^{m_{3}}(4, q-1) q^{m_{4}\left(m_{4}-1\right) / 2} \\
& \quad \times(4, q-1) q^{m_{5}\left(m_{5}-1\right) / 2}(4, q+1) q^{\left(\left(m_{6}-1\right)\left(m_{6}-2\right)+2\right) / 2}
\end{aligned}
$$

где $m_{1}+m_{2}+m_{3}+m_{4}+m_{5}+m_{6}=l, m_{4}, m_{5}, m_{6} \geqslant 2$ (некоторые из $m_{i}$ могут быть равны 0). При любых $m_{1}, m_{2}, m_{3}, m_{4}, m_{5}$ и $m_{6}$ выражение (17) не превосходит порядка большой абелевой подгруппы, указанного в таблице 2.

Пусть $\sigma_{0} \neq 1$, т.е. $O^{p^{\prime}}\left(\left(D_{l}(K)\right)_{\sigma}\right)$ - группа скрученного типа. С помощью неравенств (10)-(16) правая часть выражения (9) приводится к виду (17), но в силу леммы $4.3 m_{6} \neq 0$. Вновь при любых $m_{1}, m_{2}, m_{3}, m_{4}, m_{5}$ и $m_{6}$ выражение (17) не превосходит порядка большой абелевой подгруппы, указанного в таблице 2 .

Тип $B_{l}, q$ нечетно. В качестве следствия из леммы 4.1 получается следующий результат.

ЛЕмма 4.4 [2, предложение 11]. Пусть $G$ - групnа типа $B_{l}$ над алгебраически замкнутым полем характеристики $p \neq 2$. Пусть $G_{1}-$ редуктивная подгруппа максимального ранга группы $G$, определенная тройкой $(\lambda, \mu, \nu)$, где $\lambda, \mu-p$ азбиения, $\nu$ - ненулевое целое $u|\lambda|+|\mu|+\nu=l$. Пусть $k_{i}-$ количество частей разбиения $\lambda$, равных $i$, и $n_{i}$ - количество частей разбиения $\mu$, равных $i\left(n_{1}=0\right)$. Tогда

$$
\operatorname{Aut}_{W}\left(\Delta_{1}\right) \cong S_{k_{2}} \times\left(\mathbb{Z}_{2} \curlywedge S_{k_{3}}\right) \times\left(\mathbb{Z}_{2} \nmid S_{k_{4}}\right) \times \cdots \times\left(\mathbb{Z}_{2} \nmid S_{n_{2}}\right) \times\left(\mathbb{Z}_{2} \succ S_{n_{3}}\right) \times \cdots
$$

Пусть $G_{1}^{g}-\sigma$-инвариантная редуктивная подгруппа группы $G$, полученная скручиванием группы $G_{1}$ әлементом $w \in W$, который определяется правилом $\pi\left(g^{\sigma} g^{-1}\right)$ $=w$. Предположим, что $w$ переходит в $\tau \in \operatorname{Aut}_{W}\left(\Delta_{1}\right)$. Предположим, что $\tau$ дает пару разбиений $\xi^{(i)}, \eta^{(i)}$ с условием $\left|\xi^{(i)}\right|+\left|\eta^{(i)}\right|=k_{i}$ и пару разбиений $\zeta^{(i)}, \omega^{(i)}$ с условием $\left|\zeta^{(i)}\right|+\left|\omega^{(i)}\right|=n_{i}$ таких, что части этих разбиений дают длины положительных и отрицательных ииклов на компонентах $\tau$. Тогда простые компоненты полупростой әруппы $\left(M^{g}\right)_{\sigma}$ имеют тип

$$
A_{i-1}\left(q^{\xi_{j}^{(i)}}\right), \quad{ }^{2} A_{i-1}\left(q^{2 \eta_{j}^{(i)}}\right), \quad D_{i}\left(q^{\zeta_{j}^{(i)}}\right), \quad{ }^{2} D_{i}\left(q^{2 \omega_{j}^{(i)}}\right), \quad B_{\nu}(q)
$$

с одной компонентой для каждой части разбиений $\xi^{(i)}, \eta^{(i)} u \zeta^{(i)}, \omega^{(i)}$.

Порядок тора $\left(S^{g}\right)_{\sigma}$ дается формулой

$$
\left|\left(S^{g}\right)_{\sigma}\right|=\prod_{i, j}\left(q^{\xi_{j}^{(i)}}-1\right) \prod_{i, j}\left(q^{\eta_{j}^{(i)}}+1\right) .
$$


Из таблицы 1 и лемм 3.3, 4.4 вытекает следующее неравенство:

$$
\begin{aligned}
|A| \leqslant & 2^{l} \prod_{i, j}\left(q^{\xi_{j}^{(i)}}-1\right) \prod_{i, j}\left(q^{\eta_{j}^{(i)}}+1\right) \prod_{i, j}\left(i, q^{\xi_{j}^{(i)}}-1\right) q^{\xi_{j}^{(i)}\left[i^{2} / 4\right]} \prod_{i, j}\left(i, q^{\eta_{j}^{(i)}}+1\right) q^{\eta_{j}^{(i)}\left[i^{2} / 4\right]} \\
& \times \prod_{i, j}\left(4, q^{\zeta_{j}^{(i)}}-1\right) q^{\zeta_{j}^{(i)} i(i-1) / 2} \prod_{j}\left(4, q^{\omega_{j}^{(4)}}+1\right) q^{6 \omega_{j}^{(i)}} \\
& \times \prod_{i>5, j}\left(4, q^{\omega_{j}^{(i)}}+1\right) q^{\omega_{j}^{(i)}((i-1)(i-2)+2) / 2} 2 q^{(\nu(\nu-1)+1) / 2}
\end{aligned}
$$

С помощью неравенств (10)-(16) правая часть выражения (18) приводится к следующему виду:

$$
\begin{aligned}
& 2^{l}(2(q-1) q)^{m_{1}}(2(q+1) q)^{m_{2}}\left((3, q+1)(q+1) q^{2}\right)^{m_{3}}(4, q-1) q^{m_{4}\left(m_{4}-1\right) / 2} \\
& \quad \times(4, q+1) q^{\left(\left(m_{5}-1\right)\left(m_{5}-2\right)+2\right) / 2} 2 q^{\left(m_{6}\left(m_{6}-1\right)+1\right) / 2}
\end{aligned}
$$

где $m_{1}+m_{2}+m_{3}+m_{4}+m_{5}+m_{6}=l, m_{4}, m_{5} \geqslant 2$. При любых $m_{1}, m_{2}, m_{3}, m_{4}, m_{5}$ и $m_{6}$ выражение (19) не превосходит порядка большой абелевой подгрушшы, указанного в таблице 2 .

Групшы $B_{2}\left(2^{n}\right), n \geqslant 2$. Поскольку характеристика поля $K$ равна 2, в групше $B_{2}(K)$ нет полупростых элементов, порядок которых является плохим числом, и ее центр тривиален. Кроме того, $W\left(B_{2}\right)$ - 2-группа, поэтому $A_{0}=A$. Следовательно, центральньй тор $S$ групшы $R$ имеет размерность не менее 1 и $A \leqslant R$. Тогда возможны два случая.

Пусть $\operatorname{dim} S=1$. Тогда $R=S * A_{1}(K)$, причем $Z\left(A_{1}(K)\right)$ тривиален. Следовательно, в силу лемм 3.2 и $3.3|A| \leqslant(q+1) q \leqslant q^{3}$, т.е. не превосходит порядка большой абелевой подгруппы, указанного в таблище 2 .

Пусть $\operatorname{dim} S=2$. Тогда $R=S$ и в силу леммы $3.2|A| \leqslant(q+1)^{2}<q^{3}$, т.е. вновь меньше, чем порядок большой абелевой подгрупшы, указанньй в таблице 2 .

$T_{\text {ип }} G_{2}$. Из доказательства леммы 3.1 следует, что групша $A$ лежит в централизаторе некоторого полупростого элемента. Так как группа $G_{2}(K)$ односвязна, централизатор любого ее полупростого элемента связен (см., например, [12, теорема 2.11]). Следовательно, группа $A$ лежит в множестве неподвижных относительно морфизма Фробениуса $\sigma$ точек некоторой максимальной $\sigma$-инвариантной связной редуктивной подгруппы максимального ранга группы $G_{2}(K)$. В силу алгоритма Бореля и ди Зибенталя существуют лишь две максимальные связные редуктивные подгрупшы $R$ максимального ранга группы $G_{2}(K)$ - это групшы $A_{2}(K)$ и $A_{1}(K) * A_{1}(K)$.

Пусть $R=A_{2}(K)$. Тогда по лемме 2.2 группа $A$ содержит подгруппу $A_{0}$ индекса 3 , которая лежит в группе $A_{2}(q)$ или в ${ }^{2} A_{2}\left(q^{2}\right)$ (в силу [3, таблища 4, c. 138] возможны оба этих случая). Отметим, что оценка индекса, указанная в лемме 2.2 , довольно грубая (особенно для небольшого числа простых множителей), так как на некоторые сомножители мы делим несколько раз. Так и в нашем случае, еслигруппа $A_{2}(q)$ или ${ }^{2} A_{2}\left(q^{2}\right)$ односвязна, то она совпадает с $R_{\sigma}$ и потому подгруппа целиком лежит в $A_{2}(q)\left(\right.$ или $\left.{ }^{2} A_{2}\left(q^{2}\right)\right)$. Если же $A_{2}(q)$ (или $\left.{ }^{2} A_{2}\left(q^{2}\right)\right)$ имеет присоединенный тип, то $A$ содержит подгрупу индекса не более 3 , которая лежит в $A_{2}(q)$ (или ${ }^{2} A_{2}\left(q^{2}\right)$ ), но та, в свою очередь, имеет тривиальньй центр. Приведенные рассуждения и результаты, полученные ранее для классических групп, показьвают, что в любом случае порядок группы $A$ не превосходит одного из следующих чисел: $3 q^{2}$ или $(q+1)^{2}$, которые, очевидно, не превосходят 
числа $a\left(G_{2}(q)\right)$, указанного в таблице 2 . Далее мы будем опускать подробный разбор в аналогичных ситуациях, ссылаясь на лемму 2.2 и понимая под этим не столько само утверждение леммы, сколько метод доказательства, с помощью которого в каждом конкретном случае можно получить значительно более точную оценку, чем та, что указана в лемме.

Пусть $R=A_{1}(K) * A_{1}(K)$. Тогда в силу леммы $2.2|A| \leqslant(2, q-1) q^{2}$ либо $|A| \leqslant(q+1)^{2}$, что не превосходит числа $a\left(G_{2}(q)\right)$, указанного в таблище 2 .

Tип $F_{4}$. Поскольку групп $F_{4}(K)$ односвязна, централизатор любого полупростого элемента связен; поэтому группа $A$ содержится в некоторой собственной связной редуктивной подгруппе группы $F_{4}(K)$ максимального ранга.

В силу алгоритма Бореля и ди Зибенталя максимальные по включению собственные $\sigma$-инвариантные редуктивные связные подгрупшы максимального ранга группы $F_{4}(K)-$ Это

$$
B_{4}(K), \quad A_{2}(K) * A_{2}(K), \quad A_{1}(K) * A_{3}(K), \quad C_{3}(K) * A_{1}(K) .
$$

Пусть группа $A$ лежит в группе $R=B_{4}(K)$. Следовательно, по лемме $2.2 \mid R_{\sigma}$ : $O^{p^{\prime}}\left(R_{\sigma}\right) \mid \leqslant 2$. Таким образом, группа $A$ содержит подгруппу индекса не более чем 2 , которая лежит в группе $B_{4}(q)$. Поскольку число $a\left(B_{4}(q)\right)$ уже найдено, имеем $|A| \leqslant 2 q^{7}$, если $q$ нечетно, и $|A| \leqslant q^{10}$, если $q$ четно. В обоих случаях порядок групшы $A$ не превосходит нижней оценки числа $a\left(F_{4}(q)\right)$.

Пусть теперь группа $A$ лежит в группе $R=A_{1}(K) * C_{3}(K)$. Тогда из леммы 2.2 следует, что $|A| \leqslant 4 q^{7}$, т.е. порядок групшы $A$ менъше нижней оценки числа $a\left(F_{4}(q)\right)$.

Пусть $A$ лежит в группе типа $R=A_{1}(K) * A_{3}(K)$. Тогда $O^{p^{\prime}}\left(R_{\sigma}\right)$ изоморфна одной из следующих групп $A_{1}(q) * A_{3}(q)$ или $A_{1}(q) *{ }^{2} A_{3}\left(q^{2}\right)$ (из [3, таблица 2, c. 133] следует, что могут быть выполнены оба случая). Отсюда (вновь с использованием леммы 2.2 и известных результатов для классических групп) следует, что $|A| \leqslant 8 q^{5}$, что меньше, чем нижняя оценка числа $a\left(F_{4}(q)\right)$.

Пусть $A$ лежит в групше типа $R=A_{2}(K) * A_{2}(K)$. Тогда $O^{p^{\prime}}\left(R_{\sigma}\right)$ изоморфна группе $A_{2}(q) * A_{2}(q)$, либо ${ }^{2} A_{2}\left(q^{2}\right) *{ }^{2} A_{2}\left(q^{2}\right)$ (вновь пользуемся информацией из [3, таблица 2 , c. 133]). Отсюда вытекает оценка $|A| \leqslant 9 q^{4}$, что меньше нижней оценки числа $a\left(F_{4}(q)\right)$.

Тип $E_{6}$. Хорошо известно [21, лекция Ивахори, предложение 5], что для любого полупростого элемента $s$ из связной простой алгебраической группы $G$ группа $C_{G}(s) /$ $C_{G}^{0}(s)$ изоморфно вкладьвается в группу $\Gamma_{s c} / \Gamma_{\pi}=\Omega_{\pi}$. Поскольку для корневой системы типа $E_{6}$ справедливо $\left|\Gamma_{s c}: \Gamma_{\pi}\right|=3$, в группе $A$ существует подгруппа $A_{0}$ индекса не более чем 3 , которая содержится в некоторой собственной связной редуктивной подгруппе групшы $E_{6}(K)$ максимального ранга. В $E_{6}(K)$ существуют следующие максимальные по включению связные редуктивные подгрупшы максимального ранга:

$$
A_{1}(K) * A_{5}(K), \quad A_{2}(K) * A_{2}(K) * A_{2}(K), \quad S * D_{5}(K) .
$$

Пусть $A_{0}$ лежит в групе $R=A_{1}(K) * A_{5}(K)$. Тогда $O^{p^{\prime}}\left(R_{\sigma}\right)=A_{1}(q) * A_{5}(q)$ (см. [3, случай $\left.\left.E_{6}(q)\right]\right)$. Тогда по лемме $2.2\left|A_{0}\right| \leqslant 12 q^{10}$, откуда $|A| \leqslant 36 q^{10}$, что меньше нижней оценки числа $a\left(E_{6}(q)\right)$.

Пусть $A_{0}$ лежит в групше $R=A_{2}(K) * A_{2}(K) * A_{2}(K)$. Тогда $O^{p^{\prime}}\left(R_{\sigma}\right)$ изоморфна одной из следующих групш: $A_{2}(q) * A_{2}(q) * A_{2}(q), A_{2}\left(q^{2}\right) *{ }^{2} A_{2}\left(q^{2}\right)$ или $A_{2}\left(q^{3}\right)$ (см. [3, 
случай $\left.\left.E_{6}(q)\right]\right)$. Во всех случаях лемма 2.2 дает оценку $\left|A_{0}\right| \leqslant 27 q^{9}$ (при $q=2-9 \cdot 2^{6}$ ). Отсюда следует, что $|A|$ меньше нижней оценки числа $a\left(E_{6}(q)\right)$.

Пусть, наконец, $A_{0}$ лежит в группе $R=S * D_{5}(K)$. Тогда $O^{p^{\prime}}\left(R_{\sigma}\right)=\left(S_{\sigma}\right) * D_{5}(q)$. Из леммы 2.2 следует, что $\left|A_{0}\right| \leqslant 4(q-1) q^{10}$. Отсюда $|A|$ меньше нижней оценки числа $a\left(E_{6}(q)\right)$.

Tип ${ }^{2} E_{6}$. Индекс $\left|\Gamma_{s c}: \Gamma_{a d}\right|=3$, поэтому группа $A$ содержит подгруппу $A_{0}$ индекса не более 3 , которая лежит в некоторой собственной связной $\sigma$-инвариантной редуктивной подгрупе $R$ группы $E_{6}(K)$ максимального ранга. Максимальньми $\sigma$-инвариантными связными редуктивными подгрупшами группы $E_{6}(K)$ в этом случае будут группы

$$
A_{1}(K) * A_{5}(K), \quad S * D_{5}(K), \quad A_{2}(K) * A_{2}(K) * A_{2}(K) .
$$

Если характеристика поля $K$ четна, то в $E_{6}(K)$ не существует полупростого элемента, централизатор которого совпадал бы с $A_{1}(K) * A_{5}(K)$, в этом случае необходимо рассмотреть две дополнительные связные редуктивные $\sigma$-инвариантные подгруппы максимального ранга группы $E_{6}(q)$ - это группы $A_{5}(K) * S$ и $A_{4}(K) * A_{1}(K) * S$.

Пусть группа $A_{0}$ лежит в $R=A_{1}(K) * A_{5}(K)$ (напомним, что в этом случае $q$ нечетно). Тогда $O^{p^{\prime}}\left(R_{\sigma}\right)=A_{1}(q) *^{2} A_{5}\left(q^{2}\right)$ (см. [3, таблица 1, с. 127]). В силу леммы 2.2 $|A| \leqslant 24 q^{10}$, что меньше нижней оценки для $a\left({ }^{2} E_{6}\left(q^{2}\right)\right)$ при $q>3$. При $q=3$ замечаем, что группу $E_{6}(K)$ можно взять односвязной (так как групш ${ }^{2} E_{6}\left(3^{2}\right)_{s c}$ и ${ }^{2} E_{6}\left(3^{2}\right)_{a d}$ в этом случае изоморфны). Поэтому группа $A$ содержится в $R$ (а не ее подгруппа индекса 3$)$. Кроме того, лемма 2.2 в данном случае дает оценку $|A| \leqslant 4 q^{10}$, что меньше нижней оценки для $a\left({ }^{2} E_{6}\left(q^{2}\right)\right)$.

Пусть группа $A_{0}$ лежит в $R=S * D_{5}(K)$. Тогда $O^{p^{\prime}}\left(R_{\sigma}\right)=\left(S_{\sigma}\right) *{ }^{2} D_{5}\left(q^{2}\right)$ (см. [3, таблица 1 , с. 127]). Следовательно, $|A| \leqslant 12(q+1) q^{8}\left(|A| \leqslant 3 q^{8}\right.$, если $q$ четно), т.е. порядок групшы $A$ не превосходит нижней оценки числа $a\left({ }^{2} E_{6}\left(q^{2}\right)\right)$.

Пусть группа $A_{0}$ лежит в $R=A_{2}(K) * A_{2}(K) * A_{2}(K)$. Тогда $O^{p^{\prime}}\left(R_{\sigma}\right)$ совпадает с одной из следующих групп $A_{2}\left(q^{2}\right) * A_{2}(q),{ }^{2} A_{2}\left(q^{6}\right)$, либо с ${ }^{2} A_{2}\left(q^{2}\right) *{ }^{2} A_{2}\left(q^{2}\right) *{ }^{2} A_{2}\left(q^{2}\right)$ (см. [3, таблища 1, с. 127$])$. В любом случае справедлива оценка $|A| \leqslant 27 q^{6}$, что меньше, чем нижняя оценка для $a\left({ }^{2} E_{6}\left(q^{2}\right)\right)$.

Пусть группа $A_{0}$ лежит в $R=A_{5}(K) * S$ (этот случай мы рассматриваем лишь для поля $K$ характеристики 2). Тогда $O^{p^{\prime}}\left(R_{\sigma}\right)$ совпадает с группой ${ }^{2} A_{5}\left(q^{2}\right) * S_{\sigma}$. Отсюда следует, что порядок группы $A$ не превосходит $6(q+1) q^{9}\left(3 q^{9}\right.$, если $\left.q=2\right)$, что меньше нижней оценки числа $a\left({ }^{2} E_{6}\left(q^{2}\right)\right)$.

Пусть группа $A_{0}$ лежит в $R=A_{4}(K) * A_{1}(K) * S$ (вновь предполагается, что характеристика поля $K$ четна). Тогда $O^{p^{\prime}}\left(R_{\sigma}\right)$ совпадает с ${ }^{2} A_{4}\left(q^{2}\right) * A_{1}(q) * S_{\sigma}$, откуда $|A| \leqslant 15(q+1)^{2} q^{6}\left(|A| \leqslant 3^{2} \cdot 2^{6}\right.$, если $\left.q=2\right)$, что меньше, чем нижняя оценка для $a\left({ }^{2} E_{6}\left(q^{2}\right)\right)$.

Tип $E_{7}$. Индекс $\left|\Gamma_{s c}: \Gamma_{a d}\right|=2$, поэтому группа $A$ содержит подгрупшу $A_{0}$ индекса 2 , которая лежит в собственной связной редуктивной подгрупе $R$ группы $E_{7}(K)$. Максимальная по включению связная редуктивная подгруппа максимального ранга в $E_{7}(K)$ - это одна из следующих груп:

$$
A_{7}(K), \quad A_{1}(K) * D_{6}(K), \quad A_{1}(K) * A_{3}(K) * A_{3}(K), \quad A_{2}(K) * A_{5}(K), \quad S * E_{6}(K) .
$$

Пусть группа $A_{0}$ лежит в $R=A_{7}(K)$. Тогда $O^{p^{\prime}}\left(R_{\sigma}\right)=A_{7}(q)$ либо $O^{p^{\prime}}\left(R_{\sigma}\right)=$ ${ }^{2} A_{7}\left(q^{2}\right)$ (см. [4, таблица 1$]$ ). Из леммы 2.2 следует, что $|A| \leqslant 16 q^{16}$, т.е. порядок группы $A$ не превосходит нижней оценки числа $a\left(E_{7}(q)\right)$. 
Пусть групша $A_{0}$ лежит в $R=A_{1}(K) * D_{6}(K)$. Тогда $O^{p^{\prime}}\left(R_{\sigma}\right)$ совпадает с $A_{1}(q) * D_{6}(q)$ (см. [4, таблица 1$]$ ). В любом случае $|A| \leqslant 16 q^{16}$, что меньше нижней оценки числа $a\left(E_{7}(q)\right)$.

Пусть группа $A_{0}$ лежит в $R=A_{1}(K) * A_{3}(K) * A_{3}(K)$. Тогда $O^{p^{\prime}}\left(R_{\sigma}\right)$ изоморфна одной из следующих групп: $A_{1}(q) * A_{3}(q) * A_{3}(q)$ либо $A_{1}(q) * A_{3}\left(q^{2}\right)$ (см. [4, таблица 1]). В любом случае лемма 2.2 дает $|A| \leqslant 64 q^{9}$, что не превосходит нижней оценки числа $a\left(E_{7}(q)\right)$.

Пусть группа $A_{0}$ лежит в $A_{2}(K) * A_{5}(K)$. Тогда $O^{p^{\prime}}\left(R_{\sigma}\right)$ изоморфна одной из следующих групп: $A_{2}(q) * A_{5}(q)$ либо ${ }^{2} A_{2}\left(q^{2}\right) *{ }^{2} A_{5}\left(q^{2}\right)$ (см. [4, таблица 1]). Следовательно, справедливо неравенство $|A| \leqslant 36 q^{11}<a\left(E_{7}(q)\right)$.

Пусть, наконец, группа $A$ лежит в $R=S * E_{6}(K)$. Тогда $O^{p^{\prime}}\left(R_{\sigma}\right)$ изоморфна одной из групп $\left(S_{\sigma}\right) * E_{6}(q)$ или $\left(S_{\sigma}\right) *^{2} E_{6}\left(q^{2}\right)$. В любом случае $|A| \leqslant 6(q-1) q^{20}<a\left(E_{7}(q)\right)$.

Тип $E_{8}$. Поскольку $\Gamma_{s c}=\Gamma_{a d}$, централизатор любого полупростого элемента связен, следовательно, группа $A$ содержится в некоторой собственной связной редуктивной подгруппе групшы $E_{8}(K)$ максимального ранга. Максимальная связная редуктивная подгруппа в $E_{8}(K)$ - это одна из следующих груп:

$$
\begin{gathered}
D_{8}(K), \quad A_{8}(K), \quad A_{1}(K) * A_{2}(K) * A_{5}(K), \\
A_{4}(K) * A_{4}(K), \quad A_{3}(K) * D_{5}(K), \quad A_{2}(K) * E_{6}(K), \quad A_{1}(K) * E_{7}(K) .
\end{gathered}
$$

Пусть групша $A$ лежит в $R=D_{8}(K)$. Тогда $O^{p^{\prime}}\left(R_{\sigma}\right)=D_{8}(q)$ (см. [4, таблица 2]). Из леммы 2.2 следует, что $|A| \leqslant 4 q^{27}<a\left(E_{8}(q)\right)$. Здесь и далее в качестве числа $a\left(E_{8}(q)\right)$ мы берем нижнюю оценку этого числа из таблищы 2.

Пусть грушпа $A$ лежит в $R=A_{8}(K)$. Тогда $O^{p^{\prime}}\left(R_{\sigma}\right)=A_{8}(q)$ либо $O^{p^{\prime}}\left(R_{\sigma}\right)={ }^{2} A_{8}\left(q^{2}\right)$ (см. [4, таблища 2]). Применяя лемму 2.2 , получаем $|A| \leqslant 9 q^{20}<a\left(E_{8}(q)\right)$.

Пусть группа $A$ лежит в $R=A_{1}(K) * A_{2}(K) * A_{5}(K)$. Тогда $O^{p^{\prime}}\left(R_{\sigma}\right)$ - это одна из следуюших групп: $A_{1}(q) * A_{2}(q) * A_{5}(q)$ либо $A_{1}(q) *^{2} A_{2}\left(q^{2}\right) *^{2} A_{5}\left(q^{2}\right)$ (см. [4, таблица 2]). Вновь из леммы 2.2 следует, что $|A| \leqslant 36 q^{12}<a\left(E_{8}(q)\right)$.

Пусть групша $A$ лежит в $R=A_{4}(K) * A_{4}(K)$. Тогда $O^{p^{\prime}}\left(R_{\sigma}\right)$ - это одна из следуюших групп: $A_{4}(q) * A_{4}(q),{ }^{2} A_{4}\left(q^{2}\right) *{ }^{2} A_{4}\left(q^{2}\right)$ или ${ }^{2} A_{4}\left(q^{4}\right)$ (см. [4, таблица 2]). Из леммы 2.2 вытекает, что $|A| \leqslant 25 q^{12}<a\left(E_{8}(q)\right)$.

Пусть групша $A$ лежит в $R=A_{3}(K) * D_{5}(K)$. Тогда $O^{p^{\prime}}\left(R_{\sigma}\right)$ изоморфна одной из следуюших групп $A_{3}(q) * D_{5}(q)$ или ${ }^{2} A_{3}\left(q^{2}\right) *{ }^{2} D_{5}\left(q^{2}\right)$ (см. [4, таблища 2]). Применяя лемму 2.2 , получаем $|A| \leqslant 16 q^{19}<a\left(E_{8}(q)\right)$.

Пусть грушша $A$ лежит в $R=A_{2}(K) * E_{6}(K)$. Тогда $O^{p^{\prime}}\left(R_{\sigma}\right)$ - это одна из следуюших групп: $A_{2}(q) * E_{6}(q)$ или ${ }^{2} A_{2}\left(q^{2}\right) *{ }^{2} E_{6}\left(q^{2}\right)$. Справедливо неравенство $|A| \leqslant 9 q^{22}<$ $a\left(E_{8}(q)\right)$.

Пусть, наконец, групша $A$ лежит в $R=A_{1}(K) * E_{7}(K)$. Тогда $O^{p^{\prime}}\left(R_{\sigma}\right)=A_{1}(q) * E_{7}(q)$. В силу леммы $2.2|A| \leqslant 4 q^{33}<a\left(E_{8}(q)\right)$.

Tип ${ }^{3} D_{4}$. В $D_{4}(K)$ существуют лишь две максимальные связные $\sigma$-инвариантные редуктивные подгрупш максимального ранга:

$$
A_{1}(K) * A_{1}(K) * A_{1}(K) * A_{1}(K) \quad \text { и } T * A_{2}(K),
$$

где $T$ - тор размерности 2. 
Пусть группа $A$ лежит в $R=A_{1}(K) * A_{1}(K) * A_{1}(K) * A_{1}(K)$. Тогда $O^{p^{\prime}}\left(R_{\sigma}\right)=$ $A_{1}(q) * A_{1}\left(q^{3}\right)$ (см. [3, таблица 7, с. 140$\left.]\right)$. Из леммы 2.2 следует, что $|A| \leqslant 4 q^{4}(|A| \leqslant 27$, если $q=2$, и $|A| \leqslant 2 q^{4}$, если $\left.q=3\right)$, что меньше, чем $q^{5}=a\left({ }^{3} D_{4}\left(q^{3}\right)\right)$.

Пусть группа $A$ лежит в $R=T * A_{2}(K)$. Тогда $O^{p^{\prime}}\left(R_{\sigma}\right)=\left(T_{\sigma}\right) * A_{2}(q)$ или $O^{p^{\prime}}\left(R_{\sigma}\right)=$ $\left(T_{\sigma}\right) *^{2} A_{2}\left(q^{2}\right)$ (см. [3, таблица 7, с. 140$]$ ). Из леммы 2.2 следует, что $|A| \leqslant 3\left(q^{2}+q+1\right) q^{2}$ $(|A| \leqslant 28$ при $q=2$ и $|A| \leqslant 117$ при $q=3)$, что вновь не превосходит $q^{5}=a\left({ }^{3} D_{4}\left(q^{3}\right)\right)$.

ТАБЛИЦА 2

\begin{tabular}{|c|c|c|}
\hline Групша $G$ & $a(G)$ & Источник \\
\hline$A_{n}(q)$, кроме $A_{1}(q), q$ четно и $A_{2}(q),(3, q-1)=1$ & $q^{\left[(n+1)^{2} / 4\right]}$ & {$[5]$} \\
\hline$A_{1}(q), q$ четно & $q+1$ & [17] \\
\hline$A_{2}(q),(3, q-1)=1$ & $q^{2}+q+1$ & {$[17]$} \\
\hline$B_{n}(q), n \geqslant 4, q$ нечетно & $q^{n(n-1) / 2+1}$ & [5] и $[7]$ \\
\hline$B_{3}(q), q$ нечетно & $q^{5}$ & [5] и $[7]$ \\
\hline$C_{n}(q)$, кроме $C_{2}(2)$ & $q^{n(n+1) / 2}$ & {$[5],[6]$ и $[8]$} \\
\hline$D_{n}(q)$ & $q^{n(n-1) / 2}$ & [5] и $[7]$ \\
\hline${ }^{2} A_{n}\left(q^{2}\right)$, кроме ${ }^{2} A_{2}\left(q^{2}\right),(3, q+1)=1$ и ${ }^{2} A_{3}\left(2^{2}\right)$ & $q^{\left[(n+1)^{2} / 4\right]}$ & [8] \\
\hline${ }^{2} A_{2}\left(q^{2}\right),(3, q+1)=1$ & $(q+1)^{2}$ & [] \\
\hline${ }^{2} A_{3}\left(2^{2}\right)$ & 27 & $\Gamma$ \\
\hline${ }^{2} D_{n}\left(q^{2}\right), n \geqslant 5$ & $q^{(n-1)(n-2) / 2+2}$ & {$[7]$} \\
\hline${ }^{2} D_{4}\left(q^{2}\right)$ & $q^{6}$ & {$[7]$} \\
\hline$G_{2}(q), q$ не делится на 3, кроме $G_{2}(2)$ & $q^{3}$ & {[]} \\
\hline$G_{2}(q), q$ - степень тройки & $q^{4}$ & {[]} \\
\hline$F_{4}(q), q$ четно & $q^{11} \leqslant a(G) \leqslant q^{17}$ & [] \\
\hline$F_{4}(q), q$ нечетно & $q^{9} \leqslant a(G) \leqslant q^{14}$ & [17] \\
\hline$E_{6}(q)$ & $q^{16} \leqslant a(G) \leqslant q^{20}$ & {$[17]$} \\
\hline$E_{7}(q)$ & $q^{27} \leqslant a(G) \leqslant q^{32}$ & [17] \\
\hline$E_{8}(q)$ & $q^{36} \leqslant a(G) \leqslant q^{61}$ & [17] \\
\hline${ }^{2} B_{2}\left(2^{2 n+1}\right)$ & $2^{2 n+2}$ & [19] \\
\hline${ }^{2} G_{2}(q)$ & $q^{2}$ & [20] \\
\hline${ }^{3} D_{4}\left(q^{3}\right)$ & $q^{5}$ & [ ] \\
\hline${ }^{2} E_{6}\left(q^{2}\right)$ & $q^{12} \leqslant a(G) \leqslant q^{20}$ & и [17] \\
\hline
\end{tabular}

Порядки $a(G)$ больших абелевых подгрупп или их оценки во всех простых конечных группах лиева типа, кроме ${ }^{2} F_{4}(q)$, приведены в таблице 2 . В этой таблице в графе “Источник" указано, в какой работе изучались группы соответствующего порядка. В том случае, когда порядок указан точно, известно строение и количество всех классов несопряженных больших абелевых групп. В том случае, когда указана оценка сверху и снизу, в настоящей работе доказано, что если некоторая абелева подгруппа $A$ групшы $G$ содержит нецентральньй полупростой элемент, то ее порядок меньше, чем порядок некоторой унипотентной абелевой подгруппы. Таким образом, большая абелева подгруппа во всех конечных простых группах лиева типа, кроме ${ }^{2} F_{4}(q)$, совпадает с большой полупростой или большой унипотентной абелевой подгруппой. Если центр группы 
лиева типа $G$ нетривиален, т.е. группа $G$ не является простой, то значение, указанное в таблице, необходимо умножить на порядок центра. Пустой ссылкой обозначена ссылка на результаты настоящей работы.

\section{СПИСОК ЦИТИРОВАННОЙ ЛИТЕРАТУРЫ}

[1] Carter R. W. Centralizers of semisimple elements in finite groups of Lie type // Proc. London Math. Soc. (3). 1978. V. 37. № 3. P. 491-507.

[2] Carter R.W. Centralizers of semisimple elements in the finite classical groups // Proc. London Math. Soc. (3). 1981. V. 42. № 1. P. 1-41.

[3] Deriziotis D. I. Conjugacy classes and centralizers of semisimple elements in finite groups of Lie type. Vorlesungen aus dem Fachbereich Mathematik der Universität Essen. V. 11, 1984.

[4] Deriziotis D. I. The centralizers of semisimple elements of the Chevalley groups $E_{7}$ and $E_{8}$ // Tokyo J. Math. 1983. V. 6. № 1. P. 191-216.

[5] Barry M. J. J. Large Abelian subgroups of Chevalley groups // J. Austral. Math. Soc. Ser. A. 1979. V. 27. №1. P. 59-87.

[6] Barry M. J. J., Wong W. J. Abelian 2-subgroups of finite symplectic groups in characteristic 2 // J. Austral. Math. Soc. Ser. A. 1982. V. 33. № 3. P. 345-350.

[7] Wong W. J. Abelian unipotent subgroups of finite orthogonal groups // J. Austral. Math. Soc. Ser. A. 1982. V. 32. № 2. P. 223-245.

[8] Wong W. J. Abelian unipotent subgroups of finite unitary and symplectic groups // J. Austral. Math. Soc. Ser. A. 1982. V. 33. № 3. P. 331-344.

[9] Горенстейн Д. Конечные простые группы. Введение в их классификиацию. М.: Мир, 1985.

[10] Carter R. W. Simple Groups of Lie Type. London: Wiley, 1972.

[11] Хамфри Дж. Линейные алгебраические группы. М.: Наука, 1980.

[12] Humphreys J. E. Conjugacy Classes in Semisimple Algebraic Groups. Math. Survey and Monographs. V. 43. Providence (R.I.): Amer. Math. Soc., 1995.

[13] Gorenstein D., Lyons R. The Local Structure of Finite Groups of Characteristic 2 Type. Memoirs Amer. Math. Soc. V. 276. Providence (R.I.): Amer. Math. Soc., 1983.

[14] Borel A., de Siebental J. Les sous-groupes fermés de rang maximum des groupes de Lie clos // Comment. Math. Helv. 1949. V. 23. P. 200-221.

[15] Steinberg R. Endomorphisms of Linear Algebraic Groups. Memoirs Amer. Math. Soc. V. 80. Providence (R.I.): Amer. Math. Soc., 1968.

[16] Borel A. Linear Algebraic Groups. New York: Benjamin, 1969.

[17] В довин Е. П. Порядки абелевых подгрупп в конечных простых группах // Алгебра и логика. 1999. Т. 38. № 2. С. 131-160.

[18] Кондратьев А.С. Подгруппы конечных групп Шевалле // УМН. 1986. Т. 41. № 1. C. 57-96.

[19] Suzuki M. On a class of doubly transitive groups // Ann. Math. 1962. V. 75. № 1. P. 105-145.

[20] Ward H. N. On Ree's series of simple groups // Trans. Amer. Math. Soc. 1966. V. 121. № 1. P. $62-80$.

[21] Семинар по алгебраическим группам. Сб. статей. М.: Мир, 1973.

Новосибирский государственный университет

E-mail : 321 vep@ccmath.nsu.ru

Поступило

10.06 .1998

Исправленный вариант

01.10 .2000 